\title{
STATICS AND KINETICS AT THE NEMATIC-ISOTROPIC INTERFACE: EFFECTS OF BIAXIALITY
}

\author{
V. Popa-Nita*, T. J. Sluckin and A. A. Wheeler \\ Faculty of Mathematical Studies, University of Southampton, \\ Southampton S017 1BJ, United Kingdom
}

(November 13, 1996)

\begin{abstract}
We use the Landau-de Gennes theory of a nematic liquid crystal to investigate anew aspects of the properties of the interface between the isotropic and nematic liquid crystal phases of the same fluid. The equations of the static interface have been solved, both numerically and using asymptotic analysis, with an emphasis on the inclusion of the order parameter biaxiality on the physical properties. We have compared the results of the exact solutions to the commonly used de Gennes ansatz, which assumes positive and uniform unixiality through the interface. Although the de Gennes ansatz in general gives good results, when bend and splay elastic constants dominate over the twist constants, it can lead to errors of up to $10 \%$ in the surface energy. The asymptotic analysis also shows that, by contrast with the de Gennes ansatz, the order parameter wings in the isotropic phase exhibit negative order parameter, with principal axis perpendicular to the surface. For moving interfaces, using an approximation which at this stage does not yet include hydrodynamic coupling, we have compared our results with the analogue of the de Gennes ansatz used by the present authors in an earlier paper. We find that including biaxiality leads to larger effects in the dynamic than in the static properties, and that whereas this is essentially a perturbation to the energy, the velocity of the moving interface can be significantly slowed down. The slowing down effects are strongly correlated with surface biaxiality, but both effects seem to be diminished when the isotropic phase is advancing.
\end{abstract}

PACS:64.70Md, 68.10Jy

Typeset using REVTEX 


\section{INTRODUCTION}

The surfaces of liquid crystals present interesting features from a number of points of view. From an experimental vantage point these interesting features include: anisotropic free energies, inequivalence of surface tensions and surface free energies, and a delicate susceptibility to disruption by impurities. From a theoretical point of view, they can serve as a sensitive testing ground for phase field theories [1] of moving interphase interfaces. This is the case firstly because the phase field order parameter is known to be a physically measurable quantity, and secondly because the very fluidity of liquid crystals permits sensible time scales for useful experiments.

In a previous paper [2], which we denote henceforth as I, two of us (VPN and TJS) began an investigation into the motion of an interface between a nematic liquid crystal phase and an isotropic phase away from thermal equilibrium. This paper continues that study, with the important addition of the specifically tensor properties of the liquid crystal providing new insights. Specifically we include the effects of a non-uniform director in the nematic phase, and also include the effect of a biaxial nematic order parameter close to the nematicisotropic interface. In this context we derive some new results on the effects of these factors on stationary interfaces.

We first remind the reader of the background to the problem. Nematogenic fluids consist of anisotropic molecules which usually more or less resemble rods or discs. They possess a high temperature isotropic liquid phase which does not exhibit the molecular symmetry. There is a phase transition to an anisotropic nematic liquid crystal phase at the clearing point $T_{N I}$. This phase transition is weakly first order, and thermodynamic properties in the isotropic phase threaten to diverge at another temperature $T^{*}$. The advent of the phase transition at $T_{N I}$ avoids such complications, but the weakness of the sharp phase transition causes the nematic region between $T^{*}$ and $T_{N I}$ to be rather narrow - typically of the order of a few degrees Kelvin. The temperature $T^{*}$ represents the limit of stability of the (by now metastable) isotropic phase. There are two different types of dynamical process whereby the nematic phase is generated, depending on whether it occurs above or below $T^{*}$.

For $T<T^{*}$, because the isotropic phase is thermodynamically unstable the nucleation of the nematic phase takes place through a 'phase-ordering' kinetic process [3,4]. By contrast, when $T_{N I}>T>T^{*}$, the isotropic phase is metastable. This is the region on which we concentrate in this paper, and in which, at least in principle, a moving nematic-isotropic interface can exist.

The growth of a nematic liquid crystal into its supercooled isotropic phase is an unstable process that leads to complex morphologies of the nematic-isotropic interface. Using sharpinterface (or Stefan-type) models of solidification [5,6] the classical approach involves solving equations for the free boundary that separates the growing nematic phase from the isotropic parent phase [7,8]. The phase-field model, which has been fruitfully used in studies of solidification [1], provides an alternative approach. Now the nematic-isotropic interface is modelled by a smooth transition region of finite width in the phase-field variable (or order parameter) $Q(\mathbf{r}, t)$. The two phases correspond to regions in which the values of $Q$ are those pertaining to the isotropic and nematic phases. The relevant phase-field theory in this case turns out to be just the dynamical generalisation of the familar Landau-de Gennes theory of the liquid crystal interface $[9,10]$. The interface - now a region of finite thickness - is the 
region where the order parameter varies continuously between its two equilibrium values.

We now briefly discuss the phase-field strategy for the properties of a moving interface, and compare it with the alternative free-boundary approach. In the free-boundary approach, governing partial differential equations are solved in two phases with matching boundary conditions at a moving interface. The mathematical problem is extremely hard - sufficiently so that 'free-boundary problems' form a separate class of mathematical problems in their own right. In the phase-field approach, by contrast, the order parameter attains constant values far from the interface, each of which corresponds to an order parameter local minimum of the bulk free energy. This order parameter is governed by a nonlinear reaction-diffusion (partial differential) equation of Ginzburg-Landau type, which has the advantage that it can be solved without explicitly solving equations for the motion of any free boundary. The phase-field method does, however, require calculation of physical properties on length scales appropriate to the width of the interface - a length scale which would be deemed to be zero in the free boundary approach, and which conventional ideas in continuum mechanics would regard as insignificant for physical calculation. On the other hand, one might make a virtue out of necessity, and consider the phase field approach as able to investigate other interfacial properties essentially inaccessible within a free boundary paradigm.

Under normal circumstances, the partial differential equation for the order parameter is combined with the corresponding equations for other field variables (e.g., temperature). However, for the case of growth of a nematic liquid crystal into a supercooled isotropic phase, the thermal field exhibits a characteristic boundary-layer thickness $K_{T} / v$ (where $K_{T}$ is the thermal diffusivity and $v$ is the interface velocity) which is a factor $10^{3}$ times greater than the thickness of the interface, so that the isothermal condition remains valid, and the equations may be solved isothermally at a temperature specified in the formulation of the problem.

The time-dependent Ginzburg-Landau (TDGL) equation has found wide application in the kinetic theory of phase transitions [13,14], the propagation of signals in electric circuits [15], theoretical population biology [16] and in the phase-field models for solidification [17]. In this paper we adopt a simplified dynamical model consistent with the paradigmatic Landau-de Gennes static theory of liquid crystals. This model is a tensor TDGL theory which generalises I to include orientational effects at the surface, but omits the necessary hydrodynamic coupling which has been included in a the more complete dynamical Landaude Gennes theory of Olmsted and Goldbart [18].

This paper is organised as follows. In section II we describe the basic model and give the governing equations. In section III we study solutions that correspond to a stationary planar interface and present an asymptotic analysis that yields information about biaxiality and excess free energy. The results obtained for a planar moving interface with constant velocity are presented in section IV. In section $V$ we make some concluding remarks.

\section{MODEL}

We adopt the standard characterisation [19] of the nematic order at position $\mathbf{r}$ at time $t$ in terms of the traceless symmetric second rank tensor $Q_{i j}(\mathbf{r}, t)$ (with Cartesian indices $i, j, . .=1,2,3)$. The eigenvalue of $Q_{i j}$ largest in absolute magnitude gives the degree of ori- 
entation in the preferred direction, the corresponding eigenvector identifying the preferred direction $\mathbf{n}$ (the 'director' in the uniaxial nematic case). The difference between the remaining two eigenvalues characterises the degree of biaxiality with the biaxiality axis 1 specified by the eigenvector corresponding to the second largest eigenvalue $[4,20]$. Due to its traceless, symmetric character, the order parameter tensor $Q_{i j}$ can be represented in terms of the components of the uniaxial axis $\mathbf{n}$ and biaxial axis $\mathbf{l}$ as [21],

$$
Q_{i j}=\frac{1}{2} \eta\left(3 n_{i} n_{j}-\delta_{i j}\right)+\frac{\sqrt{3}}{2} \mu\left(l_{i} l_{j}-m_{i} m_{j}\right),
$$

where $\mathbf{m}=\mathbf{n} \times \mathbf{l}$. The general biaxial state is thus specified by five parameters, which may be considered either as (a) the five independent elements of $Q_{i j}$, or as (b) the strength of uniaxial ordering $\eta$, the biaxial scalar amplitude $\mu$, two angles to specify the orientation of the director $\mathbf{n}$, and a third angle to specify the rotation angle of $\mathbf{l}$ about $\mathbf{n}$. A special case of this is the uniaxial state, for which $\mu=0$; this is specified by only three parameters: the magnitude $\eta$, and two angles to orient the director. The parameter $\mu$ permits a more general treatment of the biaxiality which occurs as a result of spatial non-uniformity in any direction not coincident with $\mathbf{n}$. Biaxiality arises essentially in this context in bulk smectic- $\mathrm{C}$ and cholesteric liquid crystals. Of particular relevance for this paper is its occurrence in the neighborhood of those planar nematic-isotropic surfaces sustaining other than homeotropic boundary conditions [22]. It is sometimes more useful to think of the tensor $\mathbf{Q}$ expressed in matrix format in its principal axis representation:

$$
\mathbf{Q}=\left(\begin{array}{ccc}
-\eta / 2+\sqrt{3} \mu / 2 & 0 & 0 \\
0 & -\eta / 2-\sqrt{3} \mu / 2 & 0 \\
0 & 0 & \eta
\end{array}\right) .
$$

Within a mesoscopic approach such as ours, the equilibrium statistical mechanics is governed by the free energy functional

$$
\mathcal{F}(Q, T)=\int_{V}\left[f_{g}(\nabla Q)+f_{b}(Q, T)\right] d V,
$$

where $V$ is the volume of the system under consideration, $T$ its temperature, $f_{b}(Q, T)$ is the bulk free energy density and $f_{g}$ is the gradient free energy density.

We consider here systems which can sustain a spontaneous uniaxial nematic phase. Symmetry considerations require that $[9]$

$$
f_{b}(Q)=a\left(T-T^{*}\right) \operatorname{Tr}\left(Q^{2}\right)-C \operatorname{Tr}\left(Q^{3}\right)+B\left[\operatorname{Tr}\left(Q^{2}\right)\right]^{2},
$$

where the variation of the coefficients $a, B$, and $C$ with temperature $T$ is assumed to be weak and will be neglected. Similar considerations show that the gradient free-energy density has the form

$$
f_{g}=\frac{1}{2} L_{1}\left(\frac{\partial Q_{i j}}{\partial x_{k}}\right)\left(\frac{\partial Q_{i j}}{\partial x_{k}}\right)+\frac{1}{2} L_{2}\left(\frac{\partial Q_{i j}}{\partial x_{j}}\right)\left(\frac{\partial Q_{i k}}{\partial x_{k}}\right)
$$

where $L_{1}$ and $L_{2}$ are elastic constants (and the Einstein summation convention is assumed). 
The quantities $L_{1}$ and $L_{2}$ are related to the Frank-Oseen elastic constants $\left(K_{11^{-}}\right.$ splay, $K_{22}$-twist and $K_{33}$-bend) by the relations $K_{11}=K_{33}=\left(9 \eta_{b}^{2} / 2\right)\left(L_{1}+L_{2} / 2\right)$ and $K_{22}=\left(9 \eta_{b}^{2} / 2\right) L_{1}$, where $\eta_{b}$ is the bulk nematic order parameter. As usual at this level of approximation, $K_{11}=K_{33}$. The degeneracy is broken by considering higher order terms in the expansion. The difference is not important in this study. However, an important parameter in this study is $\kappa=L_{2} / L_{1}$. Large $\kappa$ favours high splay and bend elastic constants, and it is worth mentioning that it is polymeric liquid crystals, rather than small-molecule liquid crystals, which are the most likely to exhibit a high splay elastic constant.

In this study, the interface will be the $x-y$ plane, and we shall suppose all quantities to be homogeneous within this plane. The only variations occur in the $z$-direction, and derivatives with respect to $z$ will be denoted by primes.

We describe the time evolution of the order parameters using the time-dependent Ginzburg-Landau (TDGL) equation appropriate to non-conserved order parameters;

$$
\beta \frac{\partial}{\partial t} Q_{i j}(\mathbf{r}, t)=-{\frac{\delta \mathcal{F}[Q]}{\delta Q_{i j}}}^{(s)}
$$

where the superscript $(s)$ indicates that the symmetric traceless part of the functional derivative is to be taken, the transport coefficient $\beta=\gamma_{1} /\left(9 \eta_{b}^{2}\right)$ and $\gamma_{1}$ is the rotational viscosity of the nematic liquid crystal. In the present work we take $\beta$ to be constant. The same equation with additional Langevin noise term is known from the theory of critical dynamics, where it is called model $\mathrm{A}$ in the classification of Hohenberg and Halperin [23]. It can also be derived more directly from a master equation approach as the most probable evolution path of the order parameter [13]. The dynamics governed by TDGL equation (4) contains an interaction between two features: a relaxational behaviour which drives the order parameter locally to one of the minima of the bulk free energy density $f_{b}(Q)$ and an opposite diffusive behaviour due to elastic effects represented by the term arising from the gradient free energy density. We note also that the dynamics given by TDGL equation (4) neglects the coupling between the order parameter and the fluid velocity [18]. The TDGL equation is derived from a free energy functional that is applicable only to an isothermal situation. The present model therefore neglects the emission of latent heat at the interface; equivalently it is presumed that the latent heat is conducted infinitely fast away to the external environment.

In this paper we primarily consider the case in which a planar interface between the isotropic and nematic bulk phases propagates with normal velocity $+v$ into the isotropic phase. We choose Cartesian axes so that the interface is perpendicular to the $z$-axis and the condition of a flat interface requires that $Q$ vary only in the $z$ direction. The orientation of the triad $\mathbf{n}, \mathbf{l}, \mathbf{m}$ then depends on only a local tilt angle $\psi=\cos ^{-1}(\mathbf{n} \cdot \mathbf{k})$ where $\mathbf{k}$ is the unit basis vector along the $z$-axis.

We introduce non-dimensional quantities (denoted by overbars) in the following way $[2,21,25]$ :

$$
\bar{\eta}=\frac{6 B}{C} \eta ; \bar{\mu}=\frac{6 B}{C} \mu ; \tau=\frac{24 a\left(T-T^{*}\right) B}{C^{2}} ; \bar{f}_{b}=\frac{24^{2}}{C^{4}} B^{3} f_{b} ; \bar{f}_{g}=\frac{24^{2}}{C^{4}} B^{3} f_{g},
$$

where $\bar{t}=t C^{3} / 96 \beta B^{2}$ and $\bar{z}=z / \xi$ with $\xi=12 B C^{-3 / 2} \sqrt{L_{1}+2 L_{2} / 3}$. To simplify notation we henceforth omit the overbars. 
Using the representation (1a) for $Q$, the bulk free energy density (2) becomes

$$
f_{b}=\tau\left(\eta^{2}+\mu^{2}\right)-2 \eta\left(\eta^{2}-3 \mu^{2}\right)+\left(\eta^{2}+\mu^{2}\right)^{2} .
$$

The nematic-isotropic phase transition occurs at a temperature $\tau=1$. Beneath this temperature $f_{b}$ has three global minima at $(\eta, \mu)=\left(\eta_{c}, 0\right)$ and $\left(-\eta_{c} / 2, \pm \sqrt{3} \eta_{c} / 2\right)$ where $\eta_{c}=3(1+\Phi) / 4$ and $\Phi=(1-8 \tau / 9)^{1 / 2}$ which represent the nematic phase. The representation of the same bulk nematic phase by three different minima reflects the freedom in choosing any one of the vectors $\mathbf{n}, \mathbf{l}, \mathbf{m}$ to be the vector associated with the maximum eigenvalue of $\mathbf{Q}$, i.e. the director. The choice of $\mathbf{n}$ as the director puts the physically relevant minimum at $(\eta, \mu)=\left(\eta_{c}, 0\right)[20,21]$. The minima become metastable for $1<\tau<9 / 8$ and do not exist for $\tau \geq 9 / 8$, the superheating limit. The isotropic fixed point $(\eta, \mu)=(0,0)$ is metastable for $0<\tau<1$ and does not occur when $\tau \leq 0$, the supercooling limit. If $\mu=-\sqrt{3} \eta$ the two eigenvalues of $\mathbf{Q}$ associated with $\mathbf{n}$ and $\mathbf{m}$ are equal and two types of ordering occur depending on the value of $\eta$. If $-1 / 2<\eta<0$ the remaining eigenvalue is larger in magnitude and so this represents a uniaxial state with the director parallel to 1 ; if $0<\eta<1 / 4$ this eigenvalue is smaller in magnitude than the other two and this represents a uniaxial ordering in which the molecules' axes are ordered preferentially into the plane containing $\mathbf{n}$ and $\mathbf{m}$, but there is no preferred orientation within that plane. In practice these phases do not occur spontaneously, unless a very unphysical definition of the order parameter is adopted. Similar remarks hold for $\mu=\sqrt{3} \eta$ and $\mu=0$, resulting from the freedom in choosing $\mathbf{l}$ or $\mathbf{m}$ as the director.

The differential equations for $\eta, \mu$ and $\psi$ that emerge from the TDGL equation, Eq.(4), are very complicated. However, as pointed out by Sen and Sullivan [21], who examined the time-independent version of these equations, a simpler, more convenient equivalent set of governing differential equations are obtained if we define the new variables, $\eta_{s}, \mu_{s}, \nu_{s}$ in terms of the elements of the order parameter tensor in the fixed Cartesian $x y z$ frame by:

$$
\mathbf{Q}=\left(\begin{array}{ccc}
-\eta_{s} / 2+\sqrt{3} \mu_{s} / 2 & \sqrt{3} \nu_{s} / 2 & 0 \\
\sqrt{3} \nu_{s} / 2 & -\eta_{s} / 2-\sqrt{3} \mu_{s} / 2 & 0 \\
0 & 0 & \eta_{s}
\end{array}\right)
$$

explicitly, in terms of $\eta, \mu$ and $\psi$, this yields:

$$
\eta_{s}=\eta-\frac{\sqrt{3}}{2}(\sqrt{3} \eta-\mu) \sin ^{2} \psi, \quad \mu_{s}=\mu+\frac{1}{2}(\sqrt{3} \eta-\mu) \sin ^{2} \psi, \quad \nu_{s}=\frac{1}{2}(\sqrt{3} \eta-\mu) \sin 2 \psi .
$$

The bulk and gradient free energies in terms of these new variables are:

$$
f_{b}=\tau\left(\eta_{s}^{2}+\mu_{s}^{2}+\nu_{s}^{2}\right)-\left(\eta_{s}+3 \mu_{s}\right)\left[2 \eta_{s}\left(\eta_{s}-\sqrt{3} \mu_{s}\right)+3 \nu_{s}^{2}\right]+\left(\eta_{s}^{2}+\mu_{s}^{2}+\nu_{s}^{2}\right)^{2},
$$

and

$$
f_{g}=\frac{1}{2}\left[\left(\eta_{s}^{\prime}\right)^{2}+\epsilon_{1}^{2}\left(\mu_{s}^{\prime}\right)^{2}+\epsilon_{2}^{2}\left(\nu_{s}^{\prime}\right)^{2}\right]
$$


where the constants $\epsilon_{1}$ and $\epsilon_{2}$ are given by

$$
\epsilon_{1}=\sqrt{\frac{3}{3+2 \kappa}}, \quad \epsilon_{2}=\sqrt{\frac{3+3 \kappa / 2}{3+2 \kappa}},
$$

where $\kappa=L_{2} / L_{1}$, are dimensionless gradient energy coefficients. We observe that providing $\kappa$ is positive then $0<\epsilon_{1}<1$ and $\sqrt{3} / 2<\epsilon_{2}<1$. The corresponding dynamical equations are:

$$
\frac{\partial \eta_{s}}{\partial t}=-\frac{\delta \mathcal{F}}{\delta \eta_{s}}, \quad \frac{\partial \mu_{s}}{\partial t}=-\frac{\delta \mathcal{F}}{\delta \mu_{s}}, \quad \frac{\partial \nu_{s}}{\partial t}=-\frac{\delta \mathcal{F}}{\delta \nu_{s}} .
$$

These equations have moving interface solutions. We shall need to determine the interface velocity, $v$, as a function of the temperature, $\tau$, as well as other imposed conditions. These conditions (a) specify $\kappa$ which fixes the equilibrium surface orientation, and (b) specify magnetic or electric fields, which fix the orientation of the director in the nematic phase far from the boundary. It is convenient in these problems to transform the time-dependent equation into a time-independent equation in a frame co-moving with the interface.

In this frame, defined by a coordinate $z^{\prime}$, given by $z^{\prime}=z-v t$, moving with the speed of the interface, we obtain on evaluating the variational derivatives, that

$$
\begin{gathered}
\eta_{s}^{\prime \prime}+v \eta_{s}^{\prime}=\frac{\partial f_{b}}{\partial \eta_{s}}=2\left[\tau+2\left(\eta_{s}^{2}+\mu_{s}^{2}+\nu_{s}^{2}\right)\right] \eta_{s}-3\left(2 \eta_{s}^{2}-2 \mu_{s}^{2}+\nu_{s}^{2}\right), \\
\epsilon_{1}^{2} \mu_{s}^{\prime \prime}+v \mu_{s}^{\prime}=\frac{\partial f_{b}}{\partial \mu_{s}}=2\left[\tau+2\left(\eta_{s}^{2}+\mu_{s}^{2}+\nu_{s}^{2}\right)\right] \mu_{s}+3\left(4 \eta_{s} \mu_{s}-\sqrt{3} \nu_{s}^{2}\right), \\
\epsilon_{2}^{2} \nu_{s}^{\prime \prime}+v \nu_{s}^{\prime}=\frac{\partial f_{b}}{\partial \nu_{s}}=2\left[\tau+2\left(\eta_{s}^{2}+\mu_{s}^{2}+\nu_{s}^{2}\right)\right] \nu_{s}-6\left(\eta_{s}+\sqrt{3} \mu_{s}\right) \nu_{s},
\end{gathered}
$$

where prime denotes differentiation with respect to $z^{\prime}$. These are the central equations in this study.

In order to solve these equations we need extra information. The bulk nematic phase $(\eta=1, \mu=0)$ occurs as $z^{\prime} \rightarrow-\infty$ and the isotropic state $(\eta=\mu=0)$ as $z^{\prime} \rightarrow+\infty$. The angle of the director in the bulk nematic phase $(\eta=1, \mu=0)$ is taken to be fixed at $\psi_{\infty}$. In order to implement these conditions, we impose the following boundary conditions:

$$
\eta_{s}^{\prime}=\mu_{s}^{\prime}=\nu_{s}^{\prime}=0, \text { as } z^{\prime} \rightarrow-\infty, \text { and } \eta_{s}^{\prime}=\mu_{s}^{\prime}=\nu_{s}^{\prime}=0, \text { as } z^{\prime} \rightarrow \infty .
$$

We seek nontrivial solutions of equations (8), (9) and (10) and the associated boundary conditions, Eq. (11). This is a nonlinear eigenvalue problem for the order parameters $\eta_{s}, \mu_{s}, \nu_{s}$ and the temperature $\tau$ for fixed values of $\epsilon_{1}, \epsilon_{2}$ and the interface velocity $v$.

In order to solve these equations, we have integrated them numerically. We used a second-order finite-difference discretisation of the derivatives on a uniform mesh, imposing an integral constraint on $\eta_{s}$ in order to eliminate the degeneracy that results from the translational invariance of these equations and to ensure a nontrivial solution. The integral constraint was imposed using Simpson's rule. This discretisation procedure was allied to a modified Powell hybrid method, implemented in the SNSQE software [24] to solve the resulting nonlinear system of equations. Results from this procedure are given in subsequent sections. 


\section{STATIONARY INTERFACE}

\section{A. Previous theoretical work}

In this section we prepare the ground for the equations for the moving interface by studying their solutions at equilibrium, when the interface velocity is zero. It will be useful to review what is already known about the director structure of the nematic-isotropic interface. In his seminal paper a quarter of a century ago, de Gennes [9] discussed the nematicisotropic interface as the premier example of his Landau-type theory of inhomogeneous liquid crystals. On physical grounds de Gennes guessed that the biaxiality at such an interface would be small, and so he restricted consideration to a manifold of uniaxial states, with uniform director, but of course inhomogeneous principal order parameter. The resulting Euler-Lagrange equations - which correspond to a variational calculation with a constrained order parameter - are exactly soluble. As a result, de Gennes was able to calculate the angular dependence of the surface free energy; in modern parlance, we would say that he claimed to calculate the anchoring energy at the isotropic-nematic interface.

However, it is important to bear in mind that de Gennes made several mathematical leaps based on his physical intuition. The difficulty of matching mesoscopic theory (in which an order parameter profile and total energy are calculated) to its continuum limit (in which an anchoring energy and a bulk elastic energy are posited) is well-known [25-27]. The angular dependent anchoring energies do not correspond, at least as yet, to any well-defined procedure leading from solutions of Euler-Lagrange equations of the exact Landau-de Gennes free energy functional. This is an interesting and profound problem which deserves further study.

A number of remarks are in order as general background to a study of this problem. Firstly, the nematic-isotropic interface and the structure of a nematic near a wall has also recently been studied by other more microscopic methods, both in polymers [28], and in small molecule liquid crystals [29]. Secondly, de Gennes' variational solution for the nematicisotropic interface only permits stable anchoring for homeotropic boundary conditions $\left(L_{2}<\right.$ $0)$ or planar boundary conditions $\left(L_{2}>0\right)$. There is no possibility for oblique boundary conditions. The deep reason for this is the implicit neglect, in the formulation of the elastic theory, of interparticle potential terms with electric quadrupolar symmetry [30]. However, we shall not follow up the consequences of this neglect in this study.

In what follows we shall give a brief summary of the results of solving the relevant EulerLagrange equations and some of the consequent difficulties, in order to be able to use these solutions in a comparison between stationary and the moving interfaces on which we are concentrating in this study.

For a stationary planar nematic-isotropic interface $(\tau=1, v=0)$ equations $(8,9,10)$ are the Euler-Lagrange equations for $\eta_{s}, \mu_{s}$ and $\nu_{s}$ that minimise the free energy functional $\mathcal{F}(Q, T)$. We have obtained a series of numerical solutions of the governing equations with the boundary condition for $\eta_{s}$ in the nematic phase modified to specify $\eta_{s}$ to be $1-3\left(\sin ^{2} \psi_{\infty}\right) / 2$, the value appropriate to a chosen value of $\psi_{\infty}$. We considered a range of values of the bulk nematic orientation $\psi_{\infty}$ between 0 and $\pi / 2$. We found for $\psi_{\infty} \neq 0$ or $\pi / 2$ that $\eta_{s}$ and $\mu_{s}$ approach their bulk nematic state values with a small non-zero gradient. A similar behaviour was exhibited by the angle of the director. A linearised analysis of 
the governing equations about the bulk nematic phase confirms that linear dependence of the order parameters on $z^{\prime}$ can occur. This analysis shows that this linear dependence is a result of the degeneracy of bulk nematic state with respect to rotation of the director. Bulk solutions of the equivalent continuum system, for which the Frank constants $K_{11}=K_{33}$, similarly yield a linear dependence of $\psi$ with $z$.

Thus the only nontrivial solutions of equations $(8,9,10)$ are those in which the orientation of the director in the nematic phase is $\psi_{\infty}=0$ or $\psi_{\infty}=\pi / 2$. From now on, therefore we limit our attention to the two non-trivial cases: homeotropic alignment for which $\psi_{\infty}=0$, and planar alignment for which $\psi_{\infty}=\pi / 2$. We consider each in turn. In both cases $\nu_{s}=0$ and as a result the orientation of the director throughout the interface is constant. In addition $\tau=1$ and as a consequence of these considerations the governing equations reduce to the following system for $\eta_{s}$ and $\mu_{s}$ :

$$
\begin{gathered}
\eta_{s}^{\prime \prime}=2\left[1+2\left(\eta_{s}^{2}+\mu_{s}^{2}\right)\right] \eta_{s}-3\left(2 \eta_{s}^{2}-2 \mu_{s}^{2}\right), \\
\epsilon_{1}^{2} \mu_{s}^{\prime \prime}=2\left[1+2\left(\eta_{s}^{2}+\mu_{s}^{2}\right)\right] \mu_{s}+12 \eta_{s} \mu_{s} .
\end{gathered}
$$

Finally we make a remark about the language in which our results will be expressed. The equations have been solved in terms of $\eta_{s}$ and $\mu_{s}$. It will sometimes be more convenient to express the results, either in terms of the diagonal cartesian components of the tensor $\mathbf{Q}$ :

$$
Q_{z z}=\eta_{s} ; Q_{x x}=-\frac{1}{2} \eta_{s}+\frac{\sqrt{3}}{2} \mu_{s} ; Q_{y y}=-\frac{1}{2} \eta_{s}-\frac{\sqrt{3}}{2} \mu_{s},
$$

or in terms of $\eta$ and $\mu$.

\section{B. Homeotropic alignment: $\psi_{\infty}=0$}

In this case we know on symmetry grounds that $\mu_{s}\left(z^{\prime}\right)=\mu\left(z^{\prime}\right)=0$; the solution is everywhere uniaxial. The standard treatment of de Gennes [9] is now exact. The equation in $\eta_{s}$ can be solved analytically, yielding:

$$
\eta\left(z^{\prime}\right)=\eta_{s}\left(z^{\prime}\right)=\frac{1}{2}\left(1-\tanh \frac{z^{\prime}}{\sqrt{2}}\right) .
$$

The surface tension is similarly analytically accessible, yielding in dimensionless units:

$$
\sigma=\frac{1}{3 \sqrt{2}}
$$




\section{Planar alignment: $\psi_{\infty}=\pi / 2$ : numerical results}

In this case there is no analytic solution. The point of reference for us is the de Gennes variational solution, which apart from a different coherence length carries over unchanged from the homeotropic case:

$$
\eta\left(z^{\prime}\right)=\frac{1}{2}\left(1-\tanh \frac{z^{\prime}}{\xi \sqrt{2}}\right) \text { and } \mu\left(z^{\prime}\right)=0,
$$

with

$$
\xi=\sqrt{\frac{1+\kappa / 6}{1+2 \kappa / 3}},
$$

where $\xi$ is a scaled correlation length.

The surface tension is similarly analogous to the homeotropic case:

$$
\sigma=\frac{1}{3 \sqrt{2}} \xi
$$

It will later be important for us to emphasise that this ansatz is everywhere of uniaxial form:

$$
Q_{y y}(z)=Q_{z z}(z)=-\frac{1}{2} \eta(z), \quad Q_{x x}(z)=\eta(z)
$$

In the fixed coordinate system this solution has representation:

$$
\eta_{s}=-\frac{1}{2} \eta ; \mu_{s}=\sqrt{3} \eta_{s}=\frac{\sqrt{3}}{2} \eta
$$

In Fig. 1 we display the results of numerical solutions of the governing equations for different values of $\kappa$. Because we are dealing with elements of a diagonal tensor (albeit traceless), it is more illuminating to view the results by following the behaviour of $Q_{x x}, Q_{y y}$ and $Q_{z z}$. In Fig. 2 we plot the same results in terms of the variables $\eta$ and $\mu$ and compare them to the de Gennes ansatz. In Fig. 3 we plot the energy density of the computed solutions and compare them to the de Gennes approximation.

We now comment on noteworthy features of the solution. The energy density of the exact solution and of the de Gennes ansatz have similar features, and as a result the surface energy is rather well described by this approximation. However some structural features of the exact solution differ markedly from the de Gennes approximation. A predominantly uniaxial structure would require that, throughout the interface, $Q_{y y} \approx Q_{z z}$. It is apparent that that this is far from being the case in the wings of the profile as it goes far into the isotropic phase. Indeed here, $Q_{y y} \approx Q_{x x}(>0)$. In this region, the order parameter is once again uniaxial, but now the principal order parameter is negative! This feature can easily be seen in Fig. 1(b), in which $\kappa=36$, much higher than a typical experimental value, but in fact remains true in Fig. 1(a), for which $\kappa=3$, is within the experimental range. 
In this region, the molecules try to avoid the plane normal to the surface, but are otherwise neutral; at any rate they do not explicitly prefer the $x$-direction. The cross-over to the negative order parameter region takes place on the isotropic side of the profile. The smaller the value of $\epsilon_{1}$ (and thus the larger the ratio $\kappa=L_{2} / L_{1}$ ), the closer this feature is to the centre of the interface profile.

Note that, interestingly, in the nematic region $Q_{y y}<Q_{z z}$. There is then a cross-over close to the centre of the order parameter profile, while $Q_{z z}$ remains negative, but $Q_{y y}$ now changes sign. In Fig. 2 we have shown the equivalent result for the biaxiality $\mu \sim\left(Q_{z z}-Q_{y y}\right)$, which changes sign near the centre of the profile, and shows a rich structure. The main structure is more or less independent of $\kappa$, though the actual magnitude, which is zero for $\kappa=0$, seems to increase with $\kappa$.

The surface energy is relatively unaffected by the relaxation of the uniaxiality condition; this is because the interesting structural features take place on the isotropic side of the profile. This is further evidenced in Fig. 3 which shows that the most pronounced (albeit small) deviation of the free energy density from that given by the de Gennes ansatz occurs in the isotropic side of the interface.

We show in Fig. 4 how the surface energy differs from its de Gennes value, as a function of $\kappa$. A common physical value of $\kappa$ is between 2 and 4 . As $\kappa$ is increased the difference between the calculated and the de Gennes surface tension increases, though even for very large $\kappa$ this difference is never more than about $10 \%$. Large values of $\kappa$ also correspond to a heightened anomalous order parameter structure.

Having observed the main features of the order parameter profiles through the static nematic-isotropic interface, we now interpret them, firstly from a mathematical, and subsequently from a physical standpoint.

\section{Planar alignment: asymptotic analysis}

It may be shown that the governing equations possess an asymptotic solution, valid in the limit $\kappa=L_{2} / L_{1} \rightarrow \infty$, which corresponds to $\epsilon_{1} \rightarrow 0$. This solution consists of three regions; two outer regions, labelled I (the nematic hinterland) and III (the isotropic hinterland), separated by an internal layer II (the immediate surface region) of thickness $\mathcal{O}\left(\epsilon_{1}^{2 / 3}\right)$. For the purposes of describing the asymptotic structure of the outer regions it is convenient to locate Region II at $z^{\prime}=0$. (However we note that in all the figures we have translated the solutions so $z^{\prime}=0$ corresponds to the maximum value of the free energy density, which is a more physically appropriate definition of the centre of the interface.)

The leading order approximations in the outer regions are given by:

Region I, The Nematic Hinterland, $z^{\prime}<0$ :

$$
\begin{gathered}
\eta_{s}=-\frac{1}{8}\left[1+3 \tanh ^{2}\left(\alpha z^{\prime}+z^{\star}\right)\right] ; \\
\mu_{s}=\frac{\sqrt{3}}{8} \sqrt{-3+22 \tanh ^{2}\left(\alpha z^{\prime}+z^{\star}\right)-3 \tanh ^{4}\left(\alpha z^{\prime}+z^{\star}\right)},
\end{gathered}
$$


where $\alpha=\sqrt{3 / 2}$, and $\left.z^{\star}=\tanh ^{-1}(11-4 \sqrt{7}) / 3\right)$.

Region III, The Isotropic Hinterland, $z^{\prime}>0$ :

$$
\begin{gathered}
\eta_{s}=-\frac{1}{1-\beta \exp \left(2 \sqrt{2} z^{\prime}\right)} ; \\
\mu_{s}=0
\end{gathered}
$$

where $\beta=(\sqrt{7}-5) /(\sqrt{7}-3)$. Gratifyingly, the asymptotic isotropic region indeed yields $Q_{x x}=Q_{y y}$.

While it is possible to reexpress these relations in terms of $\eta$ and $\mu$, using the relations given by Eq. (7b), it turns out not to be fruitful to do so. However, the leading order approximations to $\eta$ and $\mu$ are related in the outer regions in the following manner:

Region I, The Nematic Hinterland, $z^{\prime}<0$ :

$$
\mu=\frac{-3 \sqrt{3}+\sqrt{19+24 \eta-16 \eta^{2}}}{4}
$$

where $1>\eta>(3-\sqrt{7}) / 4$.

Region III, The Isotropic Hinterland, $z^{\prime}>0$ :

$$
\mu=-\sqrt{3} \eta
$$

where $(3-\sqrt{7}) / 4>\eta>0$. In Fig. 5 we plot $\mu$ against $\eta$ for $\epsilon_{1}=0.5774,0.2$ and 0.02. It clearly demonstrates the convergence of the numerically computed solutions to the asymptotic solution in the two outer regions as $\epsilon_{1}$ decreases to zero.

\section{E. Planar alignment: approximate solution}

In Fig. 5 we have seen that the maximum magnitude of the biaxiality throughout the interface, $|\mu|_{\max }$, is achieved in region II, where $\mu$ is negative. From the asymptotic solution we find that, to leading order, $|\mu|_{\max }=\sqrt{3}(3-\sqrt{7}) / 4 \approx 0.153$. An examination of the behaviour of the biaxiality suggests that this is an upper bound for the magnitude of the biaxiality, independent of the value of $\epsilon_{1}$. We recall that in region III, because $\mu=-\sqrt{3} \eta$, the order parameter profile exhibits anomalous uniaxial behaviour with negative principal order parameter. In contrast, in region I the distribution of molecules is truly biaxial and represents a smooth spatial transition between the slightly ordered anomalous uniaxial state and the fully ordered normal nematic phase.

We now exploit the fact that $\mu$ is everywhere small to obtain an approximate solution valid for all possible values of $\epsilon_{1}$. We employ the method of successive approximations starting from the governing equations now expressed explicitly in terms of $\eta$ and $\mu$ : 


$$
\begin{gathered}
\frac{6+\kappa}{6+4 \kappa} \eta^{\prime \prime}-\frac{\kappa \sqrt{3}}{6+4 \kappa} \mu^{\prime \prime}=2 \eta-6 \eta^{2}+6 \mu^{2}+4 \eta\left(\eta^{2}+\mu^{2}\right), \\
-\frac{\kappa \sqrt{3}}{6+4 \kappa} \eta^{\prime \prime}+\frac{6+3 \kappa}{6+4 \kappa} \mu^{\prime \prime}=2 \mu+12 \mu \eta+4 \mu\left(\eta^{2}+\mu^{2}\right) .
\end{gathered}
$$

In the zeroth order approximation, the principal order parameter $\eta$ is given by the de Gennes solution Eq. (16); this is just Eq. (24) with the biaxiality $\mu=0$;

$$
\eta\left(z^{\prime}\right)=\frac{1}{2}\left[1-\tanh \left(\frac{z^{\prime}}{\sqrt{2} \xi}\right)\right] .
$$

We now insert this expression into Eq. (25) and ignore (a) nonlinear terms in $\mu$ and (b) the second derivative of $\mu$, yielding the next approximation for $\mu$ :

$$
\mu\left(z^{\prime}\right)=-\gamma \eta\left(z^{\prime}\right)\left[\frac{1-3 \eta\left(z^{\prime}\right)+2 \eta^{2}\left(z^{\prime}\right)}{1+6 \eta\left(z^{\prime}\right)+2 \eta^{2}\left(z^{\prime}\right)}\right],
$$

where

$$
\gamma=\frac{\kappa \sqrt{3}}{\kappa+6}
$$

In Fig. 6 we compare the numerical solution in $(\eta, \mu)$-space to that given by the approximation in Eq. (26). We observe that, despite the rather ad hoc nature of our approximation scheme, there is good agreement, particularly when $\mu$ is smaller. The value of this approximation is that it provides a relatively simple form that gives the correct qualitative behaviour of $\mu$. This approximation shows that the magnitude of the biaxiality increases as $\kappa=L_{2} / L_{1}$ increases, and, because $\lim _{\kappa \rightarrow \infty} \gamma=\sqrt{3}$, the magnitude of the biaxiality is bounded. An improved approximation may be obtained by retaining the nonlinear terms in $\mu$ in Eq. (25), in which case $\mu$ satisfies

$2 \mu^{3}\left(z^{\prime}\right)+\gamma\left[3+2 \eta^{2}\left(z^{\prime}\right)\right] \mu^{2}\left(z^{\prime}\right)+\mu\left(z^{\prime}\right)\left[1+6 \eta\left(z^{\prime}\right)+2 \eta^{2}\left(z^{\prime}\right)\right]+\gamma \eta\left(z^{\prime}\right)\left[1-3 \eta\left(z^{\prime}\right)+2 \eta^{2}\left(z^{\prime}\right)\right]=0$.

The appropriate root gives a better approximation for $\mu$. Although this is more complicated and hence less useful, it does have the advantage that in the limit $\kappa \rightarrow \infty$ it recovers the asymptotic solution given by Eq. (21a) and Eq. (22a).

\section{F. Anchoring Energy}

The anchoring energy $W\left(\psi_{0}\right)$, defined as the angular part of the surface energy, plays an important role in defining the boundary conditions within the macroscopic theory of the liquid crystal interfacial properties. The quantity $\psi_{0}$ is the value of the tilt angle $\psi$ at the interface. In view of its importance, an important goal of the microscopic theory of liquid crystal interfaces is the calculation of the anchoring energy. Unfortunately, as indicated above [25-27], this task has so far proved extremely difficult. The anchoring energy enters 
the macroscopic equations only when $\psi$ varies with $z$, and in this situation, it is not yet possible to provide a well-defined criterion for $\psi_{0}$.

de Gennes [9] cut through this problem by making an ansatz of constant $\psi(z)$, and then solving the resulting variational problem subject to a uniaxial profile everywhere. The result is an anchoring energy of the form (in non-dimensional units):

$$
\sigma\left(\psi_{0}\right)=\frac{1}{3 \sqrt{2}} \xi\left(\psi_{0}\right),
$$

with the angular dependent correlation length defined by:

$$
\xi\left(\psi_{0}\right)=\frac{1}{2} \sqrt{\frac{12+\kappa\left[5+3 \cos \left(2 \psi_{0}\right)\right]}{3+2 \kappa}} .
$$

This approximate form for the anchoring energy always possesses a minimum for the planar alignment for all $\kappa>0$, and always possesses a maximum at the exact, but nonequilibrium solution at $\psi_{0}=0$.

The de Gennes ansatz thus involves two elements: (a) uniaxiality, and (b) constant director angle. It is difficult to see how to improve on (b) without a more sophisticated connection between mesoscopic and macrosopic theory, but we have seen above that under equilibrium conditions at non-zero anchoring angles, the biaxiality plays an important qualitative role. In this subsection, therefore, we make an empirical improvement on the de Gennes solution, by relaxing the uniaxiality condition (a).

Using a Lagrange multiplier technique, we have minimised the free energy of the system subject now only to the constraint that the angle of the director is constant throughout the interface. We have extended our numerical procedure to solve the resulting modified EulerLagrange equations and calculated the surface energy of this constrained class of solutions as a function of the surface director, $\psi_{0}\left(=\psi_{i} n f t y\right)$. The resulting $W\left(\psi_{0}\right)$ takes into account the effect of biaxiality, and differs from the de Gennes form only for large $\kappa$. We show in Fig. 7 results for $\sigma\left(\psi_{0}\right)$ for the cases $\kappa=3$ (in the physical régime) and $\kappa=36$ (unphysically large $\kappa$ ), showing the de Gennes results for comparison.

\section{G. Commentary}

The main result of this section is that the de Gennes ansatz of a uniaxial profile for the planar alignment gives very good numerical results for the surface free energy. It differs at the most by a few per cent from the true result even when $\kappa$ is very large, and in fact in the physical region, where $\kappa \sim 3$ the difference is noticeably much less. Nevertheless physically the de Gennes ansatz misses an absolutely crucial feature of the physics. This is the form of the order parameter tail in the isotropic hinterland of the interface. In this region, the order parameter, far from having a uniaxial form with principal order parameter $Q_{x x}$ positive, is instead uniaxial with the principal order parameter, now $Q_{z z}$, negative.

Some reflection illuminates this point. The positive value of $\kappa$ favours a planar boundary condition, which involves destroying the $x-y$ symmetry of the surface. But in the isotropic wings of the profile, where the order parameter is any way small, the uniaxial state is favoured, 
but there is no preference for a broken symmetry state. The easiest way to match these two solutions is to have a negative order parameter, unbroken symmetry region, with $Q_{x x}=$ $Q_{y y}>0$, as observed. A similar effect occurs when the isotropic phase, close to $T_{N I}$, is in contact with a solid surface which favours a negative order parameter, [10].

The interfacial structure that we observe when $\kappa$ is large is not peculiar to the nematicisotropic phase transition: Braun, Cahn, McFadden and Wheeler [11] have observed similar behaviour in a Landau-Ginzburg model of an interphase boundary in an f.c.c. alloy when the surface energy anisotropy is large. This structure was first found in a cluster variation simulation of a copper-gold alloy by Kikuchi and Cahn [12].

\section{MOVING INTERFACES}

In this section we consider solutions in which the interface moves with constant velocity $v \neq 0$. In view of the findings in the previous section we confine our attention to the planar case $\psi_{\infty}=\pi / 2$.

We have already discussed this problem in I using the approximation that biaxiality be neglected. In this case there is an exact solution of the governing equations

$$
\eta=\frac{3}{8}(1+\Phi)\left[1-\tanh \left(\frac{z^{\prime}}{w_{0}}\right)\right],
$$

where $\Phi$ is related to the temperature, and $w_{0}$ sets the characteristic scale for the interface thickness:

$$
\Phi=\sqrt{1-\frac{8}{9} \tau}, \quad w_{0}=\frac{2^{5 / 2} \xi}{3(1+\Phi)},
$$

and the interface speed, $v$, is related to the temperature by

$$
v=\frac{3 \xi}{2^{3 / 2}}(\sqrt{9-8 \tau}-1)
$$

We have now solved the full governing equations numerically, allowing also for biaxiality. Some results are shown in Figs 8 and 9, in which we compare the dependence of $v$ upon $\tau$ predicted by the numerical calculations with those of the uniaxial approximation eq.(30). In Fig. 8 we show the dependence of the interfacial velocity $v$ on $\tau$ for $\kappa=4$ and $\kappa=36$. In Fig. 9 is shown the dependence of the biaxiality $\Delta \mu=\mu_{\max }-\mu_{\min }$, on $\tau$. A summary of the results is as follows. The relaxation of the uniaxiality condition slows the motion of the interface. As the degree of biaxiality depends on $\kappa$, the effect is largest for large $\kappa$. For any given value of $\kappa$, the degree of biaxiality is larger than at phase coexistence at low temperatures $(0<\tau<1)$, but smaller at higher temperatures $(1<\tau<9 / 8)$. The degree of slowing of the moving interface is positively associated with the degree of biaxiality of the interface. As a consequence the biaxiality effects are more important when the nematic phase is advancing, and particularly so when it is advancing fastest. They are almost absent when the isotropic phase is advancing. Why there should be a noticeable effect for an advancing nematic phase, but not so for an advancing isotropic phase remains a mystery. 
A particularly interesting aspect of our results concerns the magnitude of the effect. At the probably unphysically large value of $\kappa=36$, the error in the surface energy made by assuming uniaxiality is less that $10 \%$. The interface velocity, by contrast, is retarded by approximately a factor of $2 / 3$ by comparison to that that predicted by Eq. (30) for growth of the nematic phase. Thus biaxiality, although only marginally relevant to the static properties of the nematic-isotropic interface, can be of profound importance to its dynamics.

\section{CONCLUSIONS}

In this paper we have used the Landau-de Gennes theory to study both stationary and moving interfaces between a nematic liquid crystal and its own isotropic liquid phase. The principal focus of the study of the stationary interfaces has been to investigate the biaxiality, which the rather simple de Gennes ansatz does not take into account.

The biaxiality effects depend on the magnitude of the elastic constant $L_{2}$, which in turn also distinguishes splay and bend from twist in the bulk nematic. If $L_{2}$ is less than zero, the interface is homeotropic and then ipso facto, because of the unbroken surface symmetry, there is no biaxiality. If $L_{2}$ is greater than zero, the equilibrium configuration swings round into the surface plane, and now biaxiality effects increase with $L_{2}$.

Quantitatively, the effect of the biaxiality on the surface tension is always less than $10 \%$, and so the de Gennes approach seems safe for most practical purposes. Using an asymptotic analysis, however, we have found that the liquid crystal order parameter takes a very different form from the de Gennes form as it decays into the isotropic phase. Specifically the uniaxial order parameter with largest positive eigenvalue in the surface plane is transformed into a uniaxial order parameter with largest negative order parameter perpendicular to the surface plane, thus restoring the surface-induced symmetry far from the interface.

This part of our study can be extended in a number of ways. First, one might wish to include explicitly those effects which allow for an equilibrium tilted surface texture, which the Landau-de Gennes theory in its present form does not allow. Second, one might wish to examine some of the present conclusions in the light of a molecular theory of the same interface, and in particular examine the effect of the density jump at the interface which the present theory ignores. This latter effect would be particularly important for a lyotropic liquid crystal. Finally we might wish to extend the theory to other orientable interfaces, such as, for example a liquid-vapour interface, a fluid-fluid interface, or the technologically interesting case of hard walls.

The second part of our paper has concerned the properties of a moving nematic-isotropic interface. This particular subject has been much less well-studied theoretically, although it has served as an experimental paradigm for phase-phase transformation, and as such continues to give rise to puzzling phenomena.

This is the second paper in our study of dynamical phenomena. The first - I in the nomenclature of the introduction - applied current understanding of the behaviour of moving interfaces for systems with static non-conserved scalar order parameters to this problem, using the de Gennes approach which effectively scalarizes a tensor problem. In this paper we have begun a more systematic study, using equations of a non-uniform nematic developed by Olmsted and Goldbart [18]. These equations are sufficiently complex that we have chosen 
to complicate the problem in several stages. In this paper, we have only allowed for the full complexity of the order parameter. In future work we shall also allow for the interaction between the complex order parameter and hydrodynamic degrees of freedom. Eventually we shall embed this theory in a more complete description of the interfacial kinetics which allows for thermal diffusion as well.

We find that by allowing for biaxiality the velocity of a moving phase interface can be significantly slowed down, and that the effects of allowing a biaxial order parameter are much larger in dynamical phenomena than in the equilibrium case. We find also that the more complex the order parameter structure, all other things being equal, the slower the moving interface. We have also uncovered some surprising features, most notably that such effects that we have found are considerably more striking when the nematic phase is advancing into the isotropic phase than vice versa.

In future work on the dynamic properties of the nematic-isotropic interface, we shall continue with the programme adumbrated above. A particular focus of attention will be the possibility of surface instabilities - already common at moving phase interfaces - which could now be induced by the competition between different ordering effects due to anchoring energy, external fields, aligning effects of tensor thermal diffusivities, and other related phenomena

\section{ACKNOWLEDGMENTS}

VPN thanks the Leverhulme Trust for a visiting research fellowship to the University of Southampton whose hospitality is gratefully acknowledged. We are also grateful to Peter Olmsted for a number of helpful communications, for sending a copy of his unpublished Ph.D thesis, and some encouragement. TJS is sorry that, despite the trouble Olmsted went to in sending him the thesis, he has lost it again. 


\section{REFERENCES}

* Permanent address: Faculty of Physics, University of Bucharest, P.O.Box MG-11, BUCHAREST 76900, ROMANIA

[1] J. S. Langer, in Directions in Condensed Matter Physics, edited by G. Grinstein and G. Mazenco (World Scientific, Philadelphia, 1986), pp 164-186.

[2] V. Popa-Nita and T.J. Sluckin, J. Phys. II (France), 6, 873-84 (1996)

[3] A. J. Bray, Adv. Phys. 43, 357 (1994).

[4] M. Zapotocky, P. M. Goldbart and N. Goldenfeld, Phys. Rev. E51, 1216 (1995).

[5] J. S. Langer, Rev. Mod. Phys. 52, 1 (1980).

[6] B. Caroli, C. Caroli and B. Roulet, in Solids Far From Equilibrium, ed. C. Godréche, Cambridge Univ. Press. 155 (1992).

[7] J. -M. Flesselles, A. J. Simon and A. J. Libchaber, Adv. Phys. 40, 1 (1991).

[8] J. Bechhoefer and S. A. Langer, Phys. Rev. E 51, 2356 (1995)

[9] P. G. de Gennes, Mol. Cryst. Liq. Cryst. 12, 193 (1971).

[10] For a review of these models see, e. g., T. J. Sluckin and A. Poniewierski, in Fluid Interfacial Phenomena, edited by C. A. Croxton (New York, Wiley, 1986), ch. 5 and B. Jérôme, Rep. Prog. Phys. 54, 391 (1991).

[11] R. J. Braun, J. W. Cahn, G. B. McFadden and A. A. Wheeler, Anisotropy of interfaces in an ordered alloy: a mulitple order parameter model. Submitted Phil. Trans. Roy. Soc. Lond.

[12] R. Kikuchi and J. W. Cahn, Acta Metall. 27, 1337 (1979).

[13] H. Metiu, K. Kitahara and J. Ross, J. Chem. Phys. 64, 292 (1976).

[14] S. K. Chan, J. Chem. Phys. 67, 5755 (1978).

[15] A. C. Scott, Rev. Mod. Phys. 47, 487 (1975).

[16] H. Haken, Synergetics(Springer,Berlin,1983).

[17] A. A. Wheeler, W. J. Boettinger and G. B. McFadden, Phys. Rev. E47, 1893 (1993).

[18] P. D. Olmsted and P. M. Goldbart, Phys. Rev. 46, 4966 (1992).

[19] P. G de Gennes and J. Prost, The Physics of Liquid Crystals (second edition, Oxford University Press, 1993).

[20] A. J. Bray, S. Puri, R. E. Blundel and A. M. Somoza, Phys. Rev. E47, R2261 (1993).

[21] A. K. Sen and D. E. Sullivan, Phys. Rev. A35, 1391 (1987).

[22] We remind readers of the conventional language describing liquid crystal boundary conditions. For homeotropic boundary conditions the directtor is perpendicular to the interface, whereas for conical and planar boundary conditions the director is tilted with respect to the surface normal and in the surface plane, rspectively.

[23] P. C. Hohenberg and B. I. Halperin, Rev. Mod. Phys. 49 , 435 (1977).

[24] SLATEC Common Math Library, National Energy Software Center, Argonne National Laboratory, Argonne, IL, written by K. L. Hiebert, based on M. J. D. Powell, in Numerical Methods for Nonlinear Algebraic Equations ed. P. Rabinowitz (Gordon and Breach, New York, 1970).

[25] P. I. C. Teixeira, T. J. Sluckin and D. E. Sullivan, Liq. Cryst. 14, 1243 (1993).

[26] H. Yokoyama, Mol. Cryst. Liq. Cryst. 165, 265 (1988).

[27] A. Poniewierski and A. Samborski, Phys. Rev. E53, 2436 (1996) 
[28] see e.g. Z.Y. Chen and J. Noolandi, Phys. Rev. A45, 2389 (1992), S-M. Cui, O. Akcakir and Z.Y. Chen. Phys. Rev. E51, 4548 (1995), Z.Y. Chen and S-M. Cui, ibid, 52, 3876 (1995).

[29] The classic molecular study of liquid crystal interfaces is by M.M. Telo da Gama, Molec. Phys. 52, 585 (1984), and this has given rise to several more recent studies.

[30] see e.g. M.A. Osipov and T.J. Sluckin, J. Phys. (France) II 3, 793 (1993).

[31] For an interesting review of this literature, and some modern experiments which have validated theories of nematic liquid crystals in an external field, see: I. Lelidis, Transitions de phases induites par un champ electrique dans les cristaux liquides Ph.D dissertation, Université de Paris-sud (unpublished) (1994) 
FIGURES 
(a)

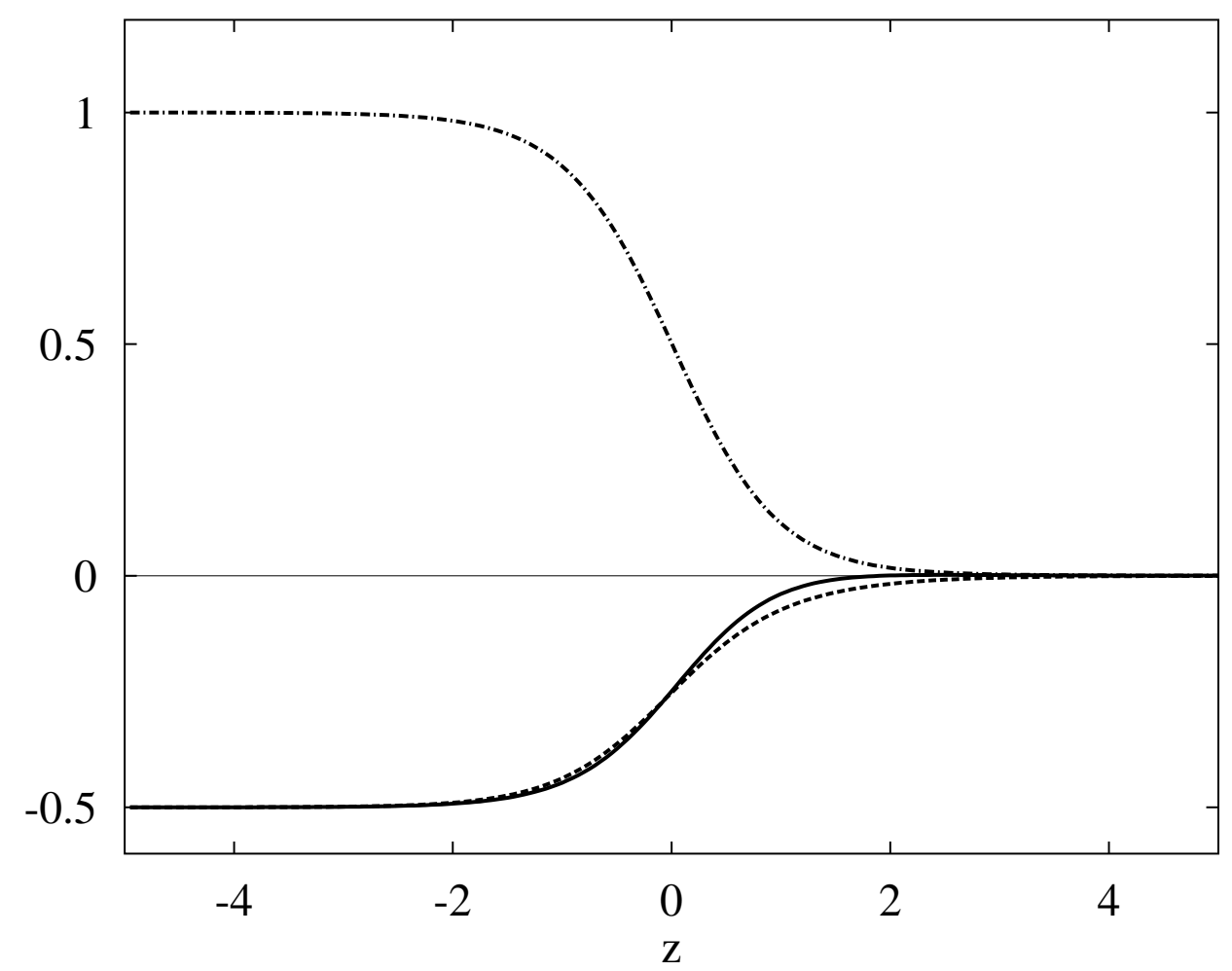

(b)

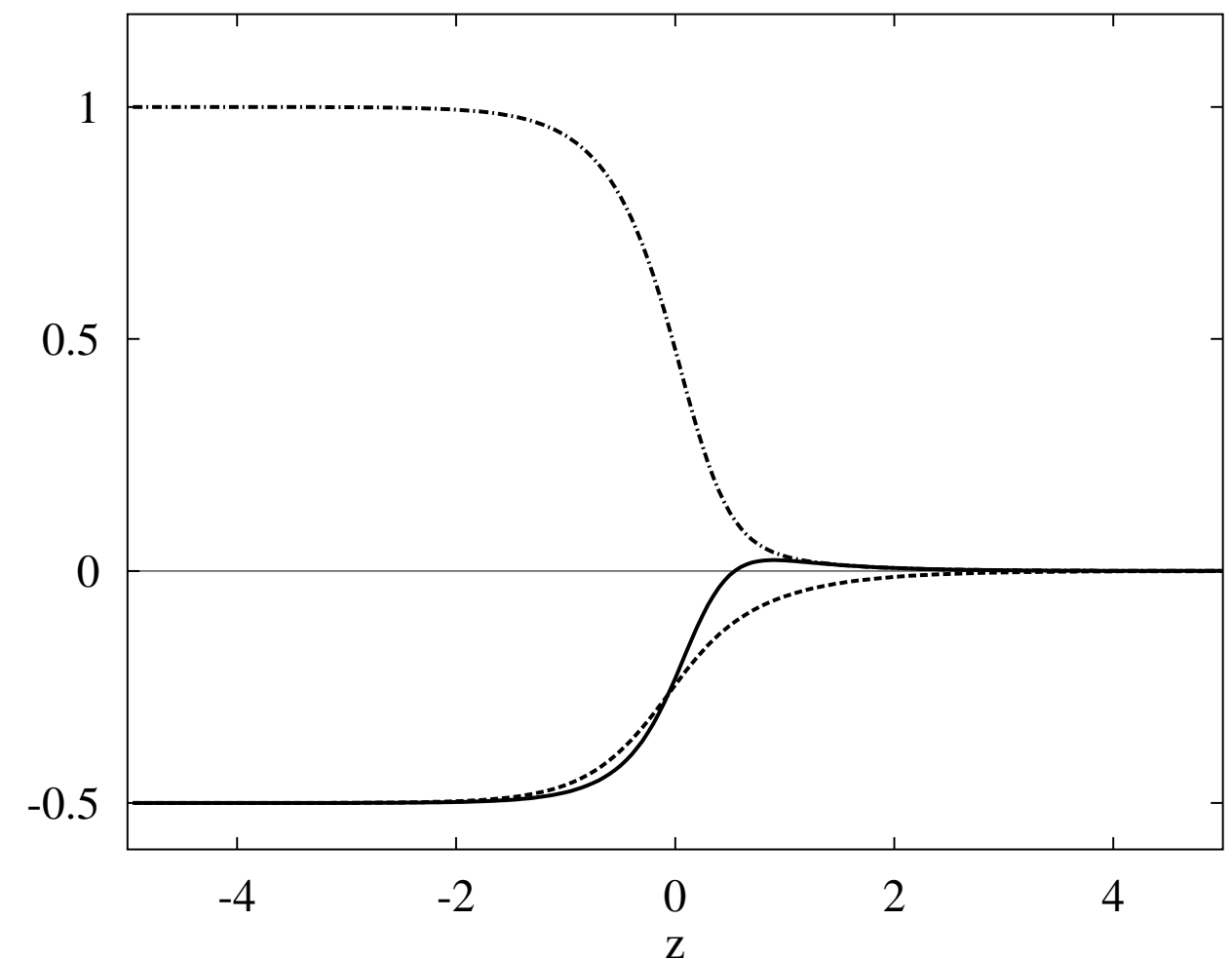

FIG. 1. Plots of the order the components of the order tensor $Q(z)$ for (a) $\kappa=3$ and (b) $\kappa=36$ obtained from direct numerical integration of equations (12). The chain-dashed curves denote $Q_{x x}(z)$, the solid curves $Q_{y y}(z)$ and the dashed curves $Q_{z z}(z)$. 
(a)

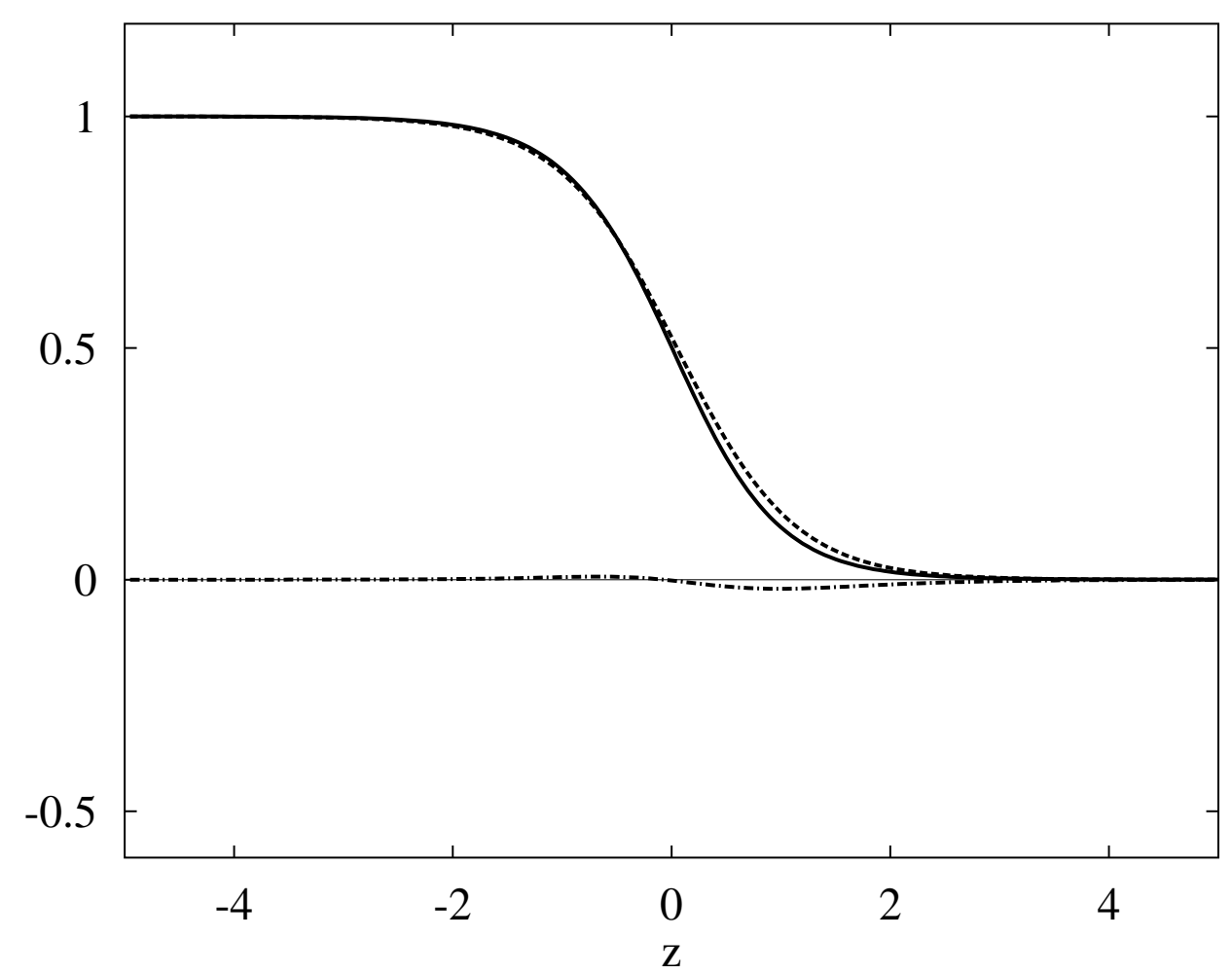

(b)

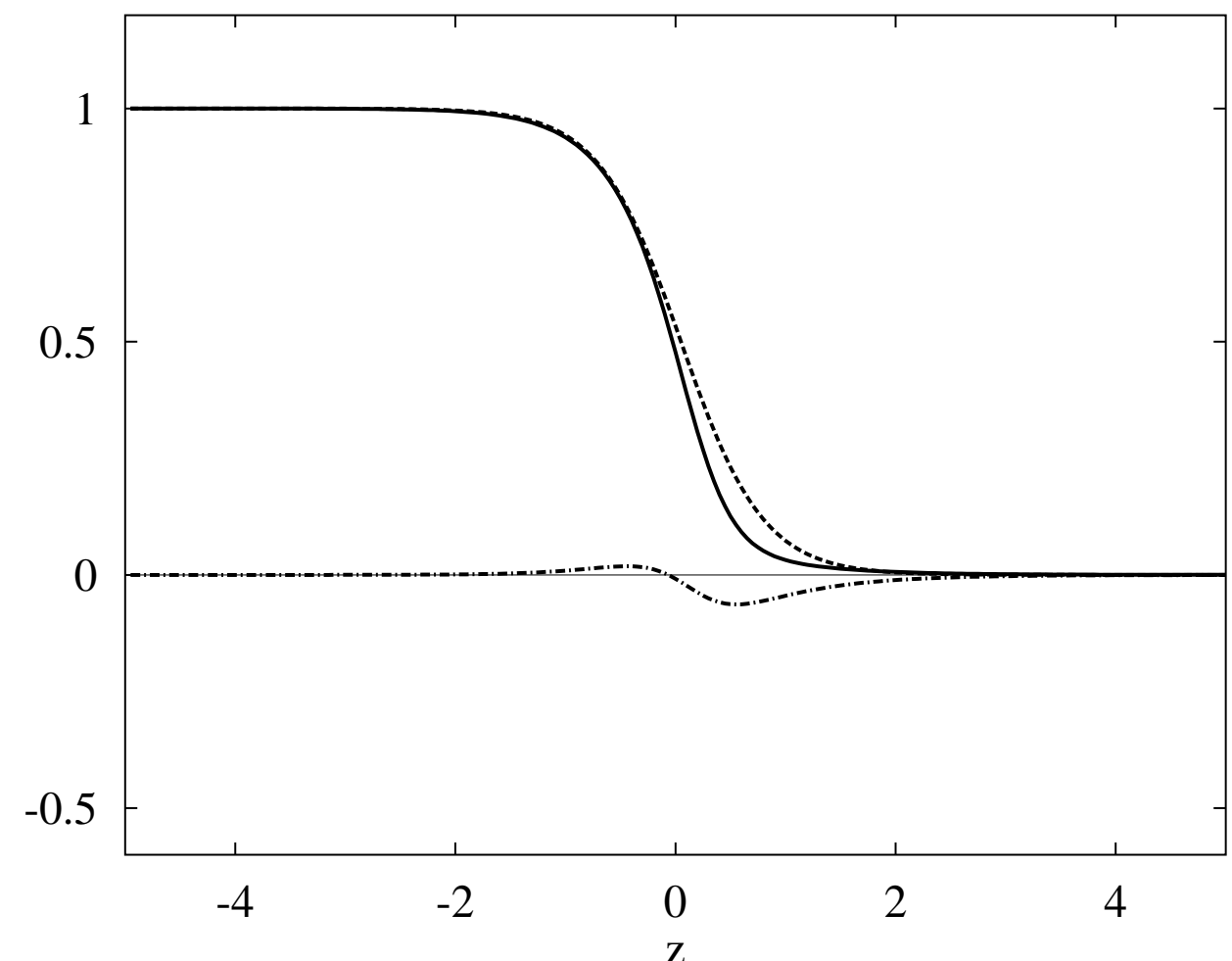

FIG. 2. Plots of $\eta(z)$ and $\mu(z)$ for (a) $\kappa=3$ and (b) $\kappa=36$. The solid and chain-dashed curves denote $\eta(z)$ and $\mu(z)$ respectively obtained from direct numerical integration of equations (12). The dashed curve is the de-Gennes approximation to $\eta(z)$ given by equation (16). 

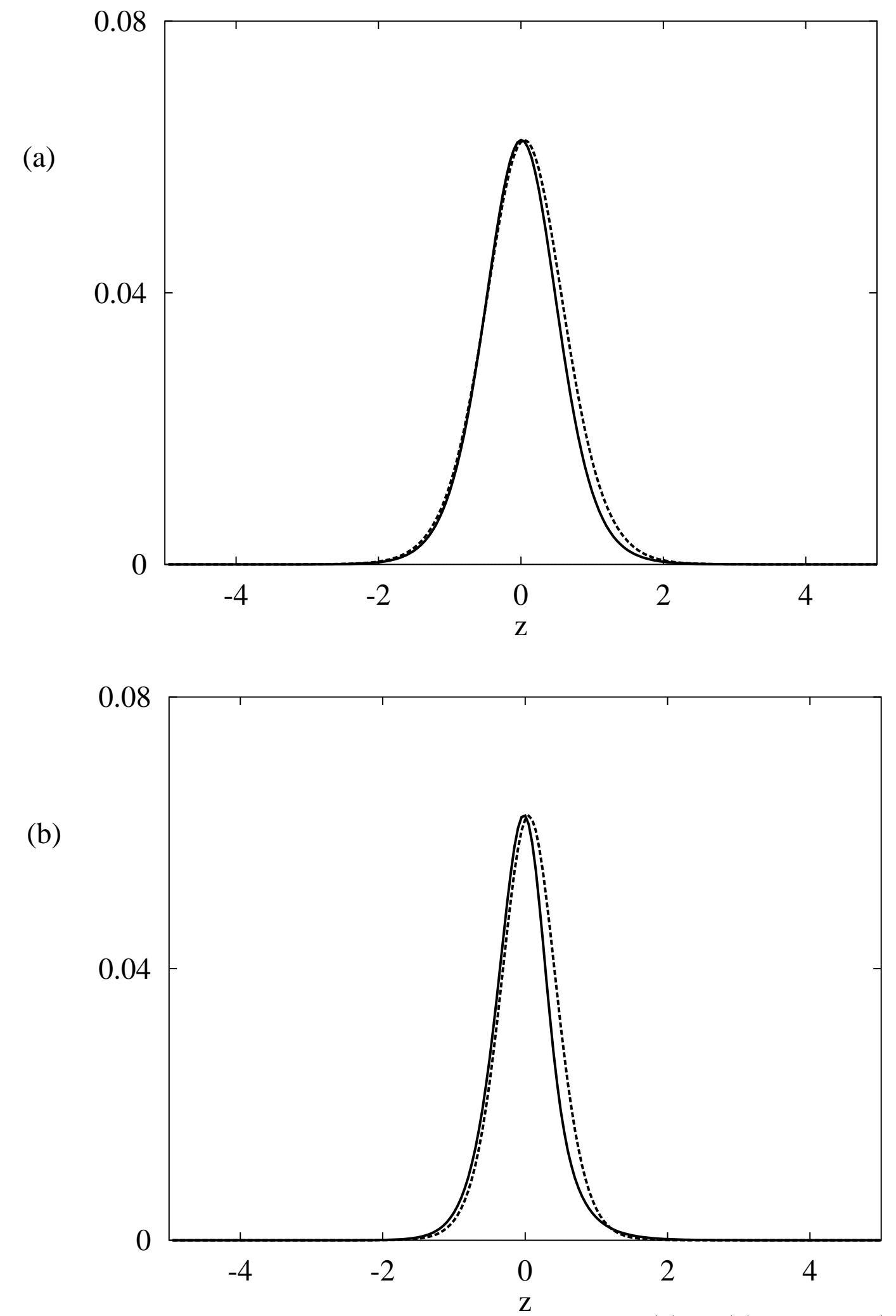

FIG. 3. The solid curves denote the bulk free energy density $f_{b}(z)$ for (a) $\kappa=3$ and (b) $\kappa=36$ derived from $\eta(z)$ and $\mu(z)$ obtained from direct numerical integration of equations (12). The dashed curves are the de-Gennes approximation to $f_{b}(z)$. 


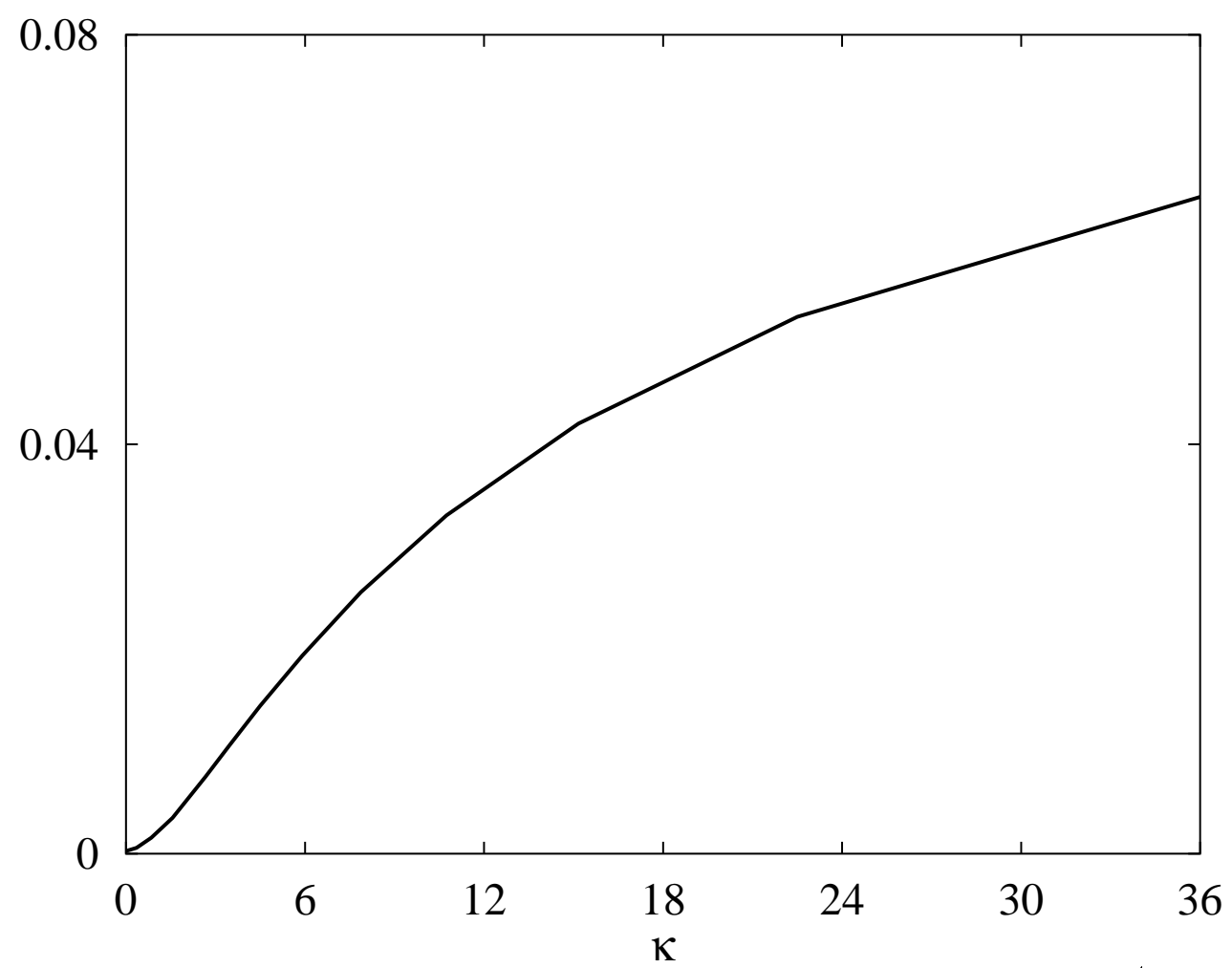

FIG. 4. To illustrate the effect of biaxiality on surface energy, $\sigma$, we show $\sigma / \sigma_{d G}-1$ as a function of $\kappa$, where $\sigma_{d G}$ is the surface energy calculated from the uniaxial form for the order parameter $Q(z)$ given by de-Gennes. 


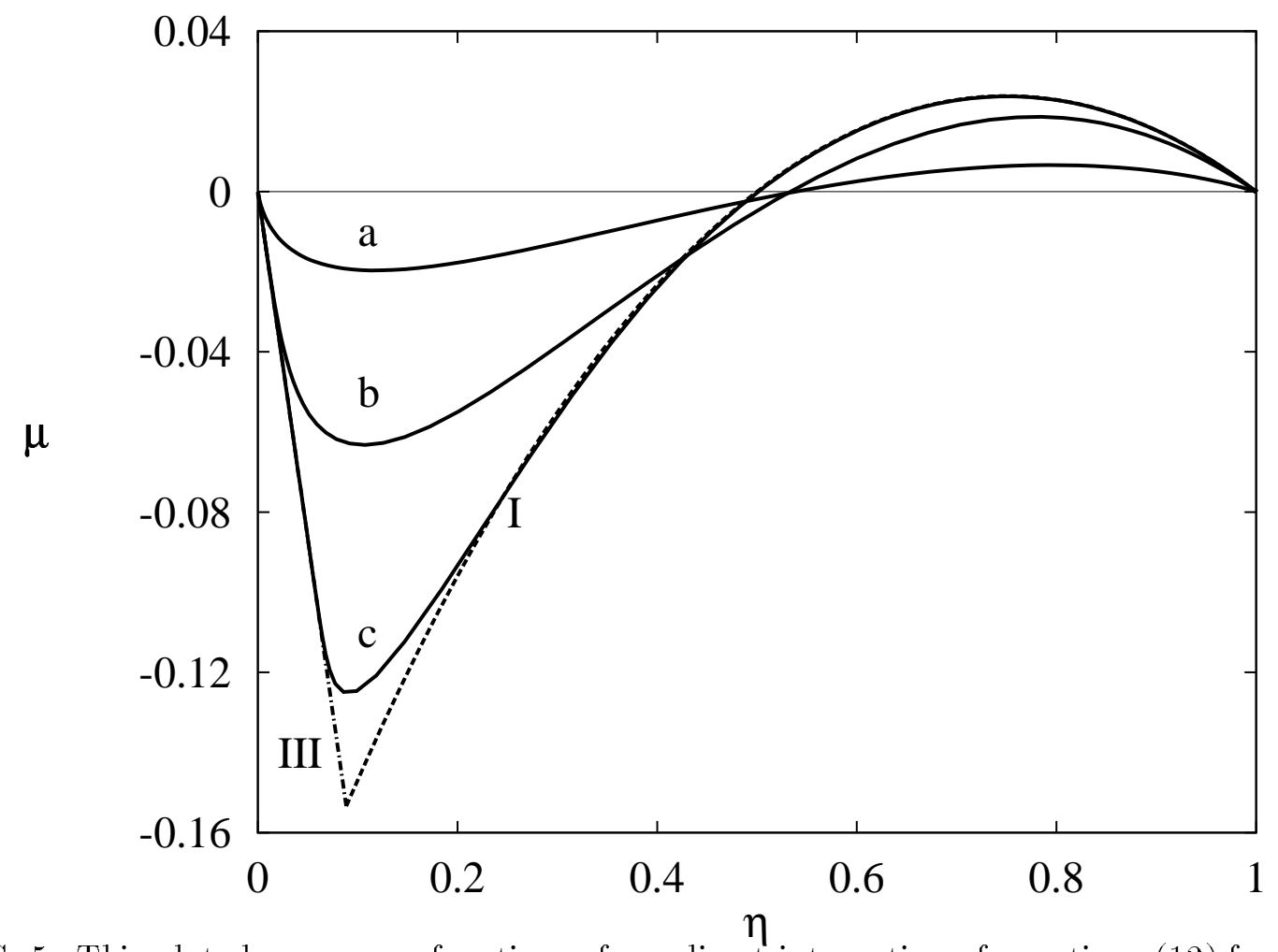

FIG. 5. This plot shows $\mu$ as a function $\eta$ from direct integration of equations (12) for a) $\kappa=3$ $\left.\left(\epsilon_{1}=0.5774\right), \mathrm{b}\right) \kappa=36\left(\epsilon_{1}=0.2\right)$ and c) $\kappa=3748.5\left(\epsilon_{1}=0.02\right)$. The dashed curves show the leading order approximations to the solution in the limit $\kappa \rightarrow \infty$ in regions I and III given by equations (23). 


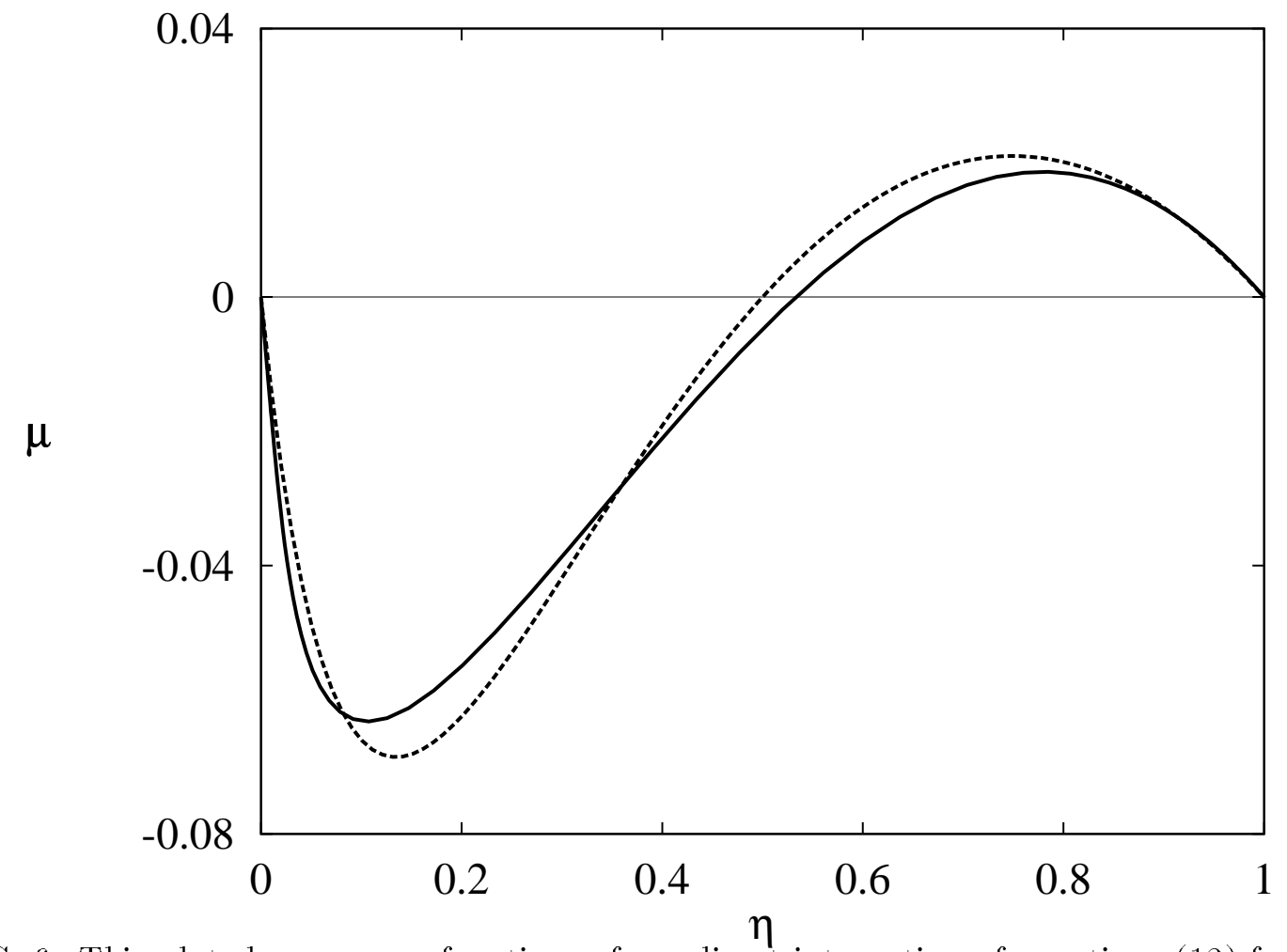

FIG. 6. This plot shows $\mu$ as a function $\eta$ from direct integration of equations (12) for $\kappa=36$. The dashed curve show the the approximation to the solution given by equation (26). 
(a)

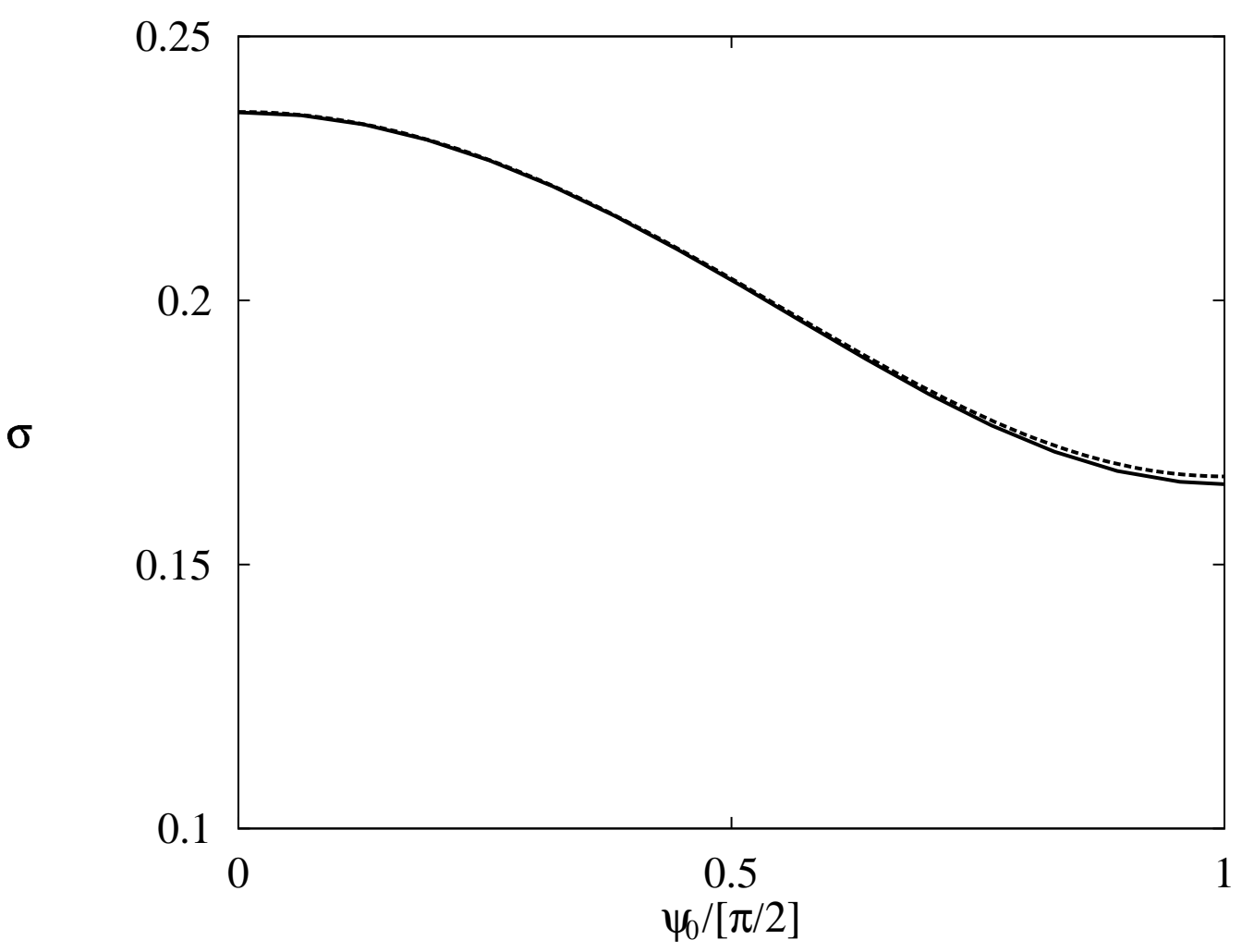

(b)

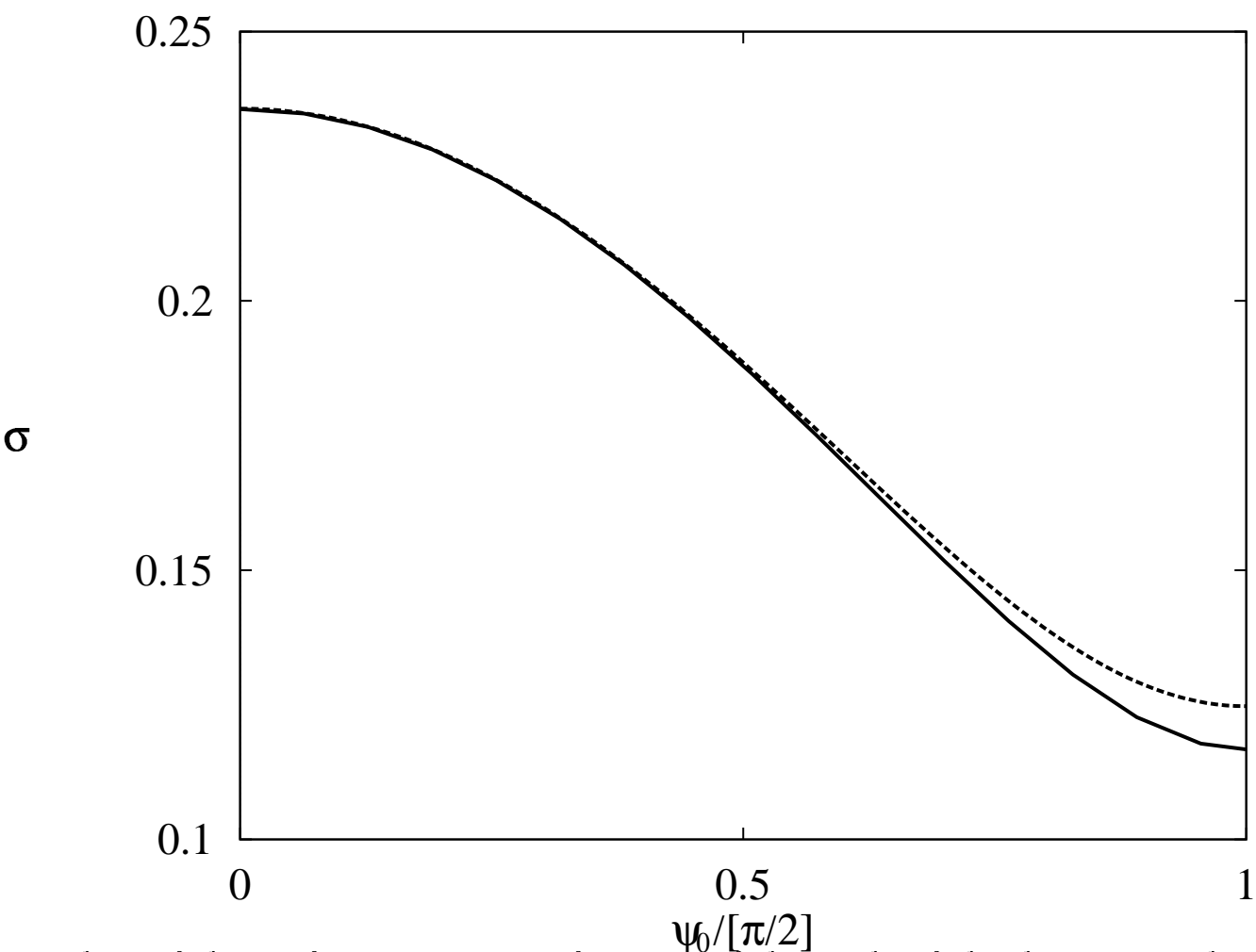

FIG. 7. Plots of the surface energy as a function of the angle of the director at the interface, $\psi_{0}$ for (a) $\kappa=3$ and (b) $\kappa=36$. The solid curves denote the results of minimising the free energy subject to the constraint that the angle director through the interface $\psi$ is constant and equal to $\psi_{0}$ (and not assuming that the biaxiality is zero). The dashed curves denote the the surface energy dependence on $\psi_{0}$ from equation (27) given by de-Gennes in which the biaxiality is neglected. 
(a)

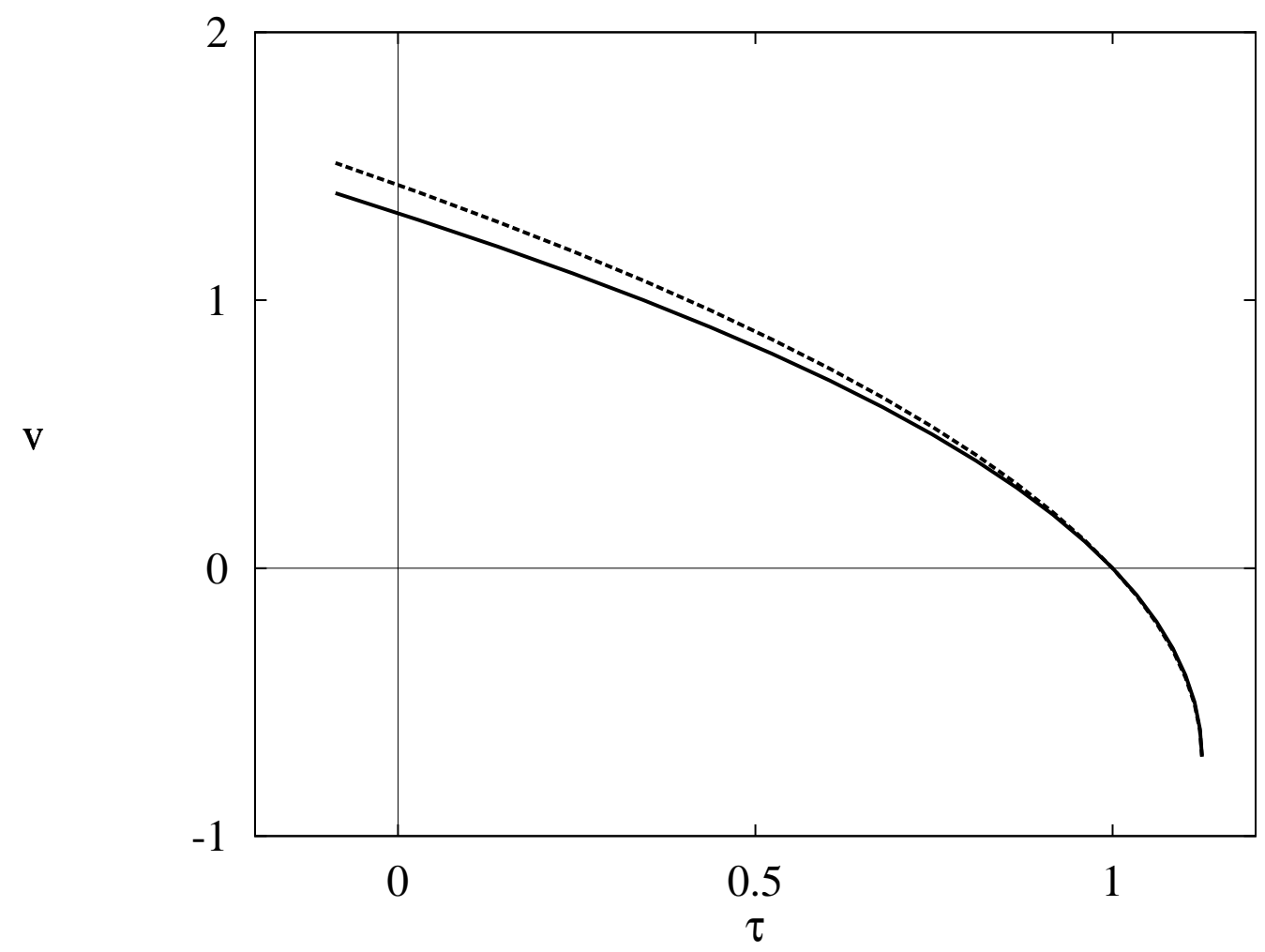

(b)

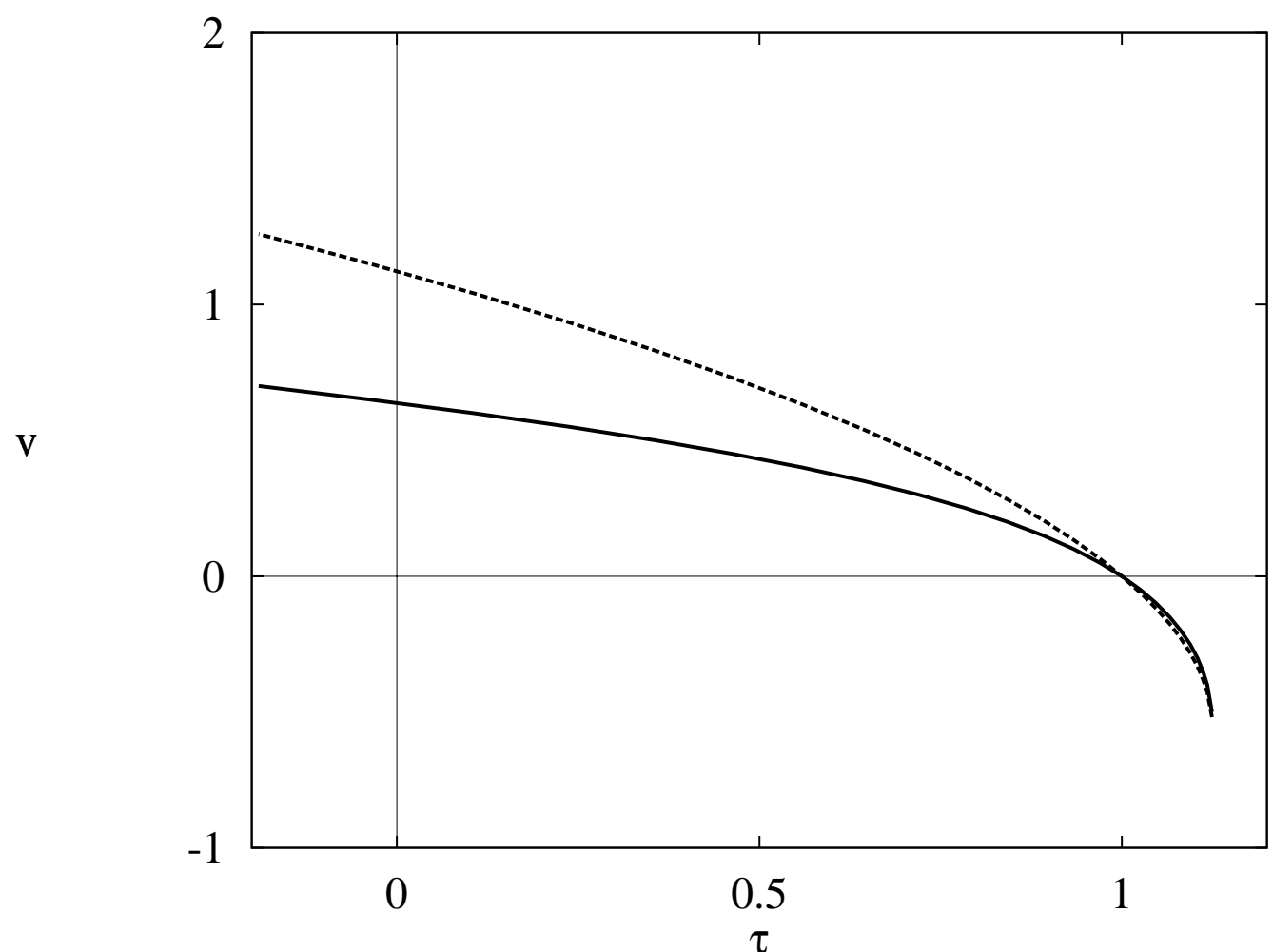

FIG. 8. Plots of the interface velocity, $v$, as a function of the interface temperature, $\tau$, for (a) $\kappa=4$ and (b) $\kappa=36$. The solid curves denote the results of numerical integration of the governing equations (8)-(10) for the case of planar alignment. The dashed curve is $v(\tau)$ predicted by equation (30), first given in I, in which biaxiality is neglected. 


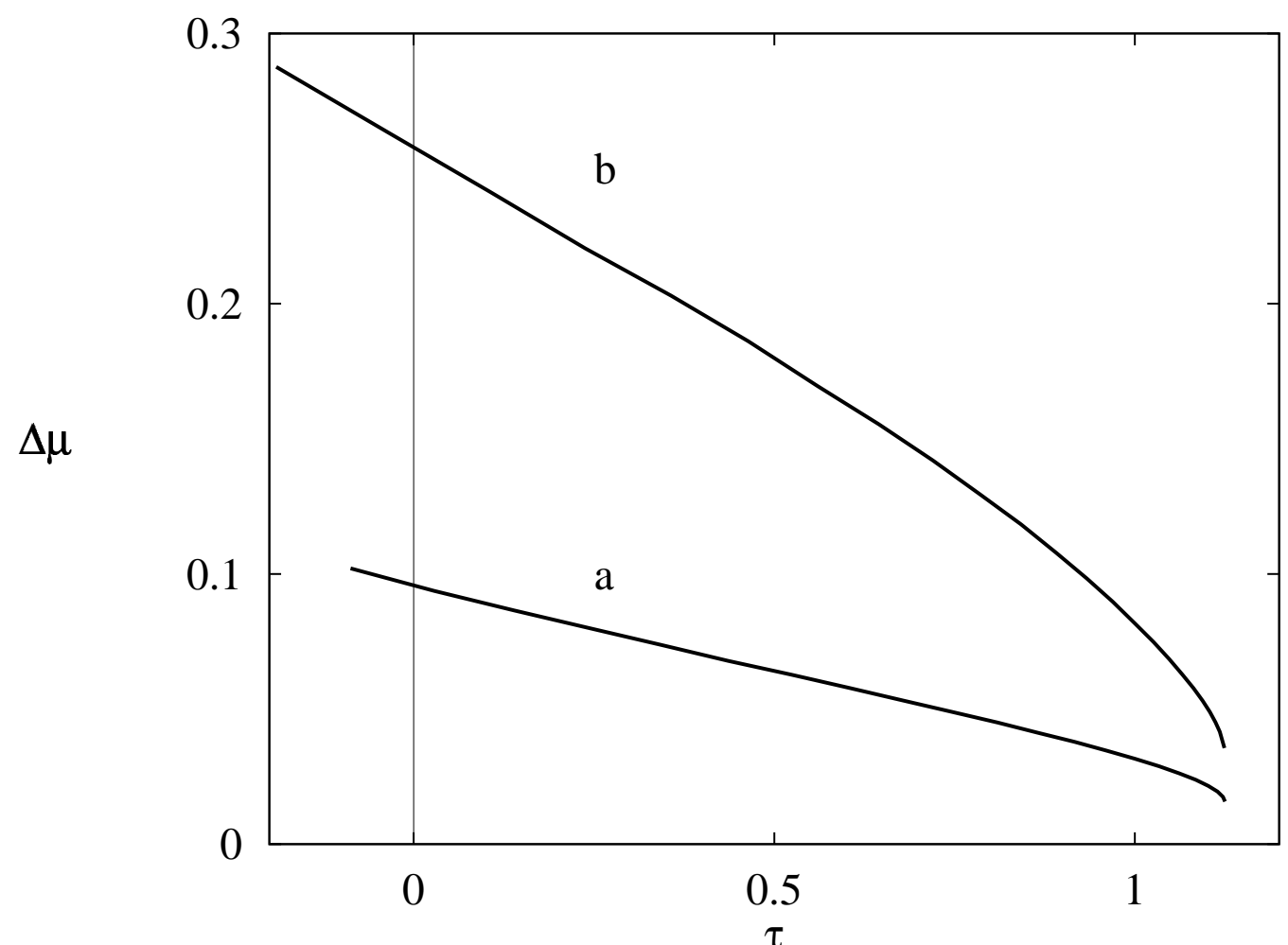

FIG. 9. This plot shows dependence of the magnitude of the biaxiality $\Delta \mu\left(=\mu_{\max }-\mu_{\min }\right)$ on temperature $\tau$ for (a) $\kappa=4$ and (b) $\kappa=36$. 